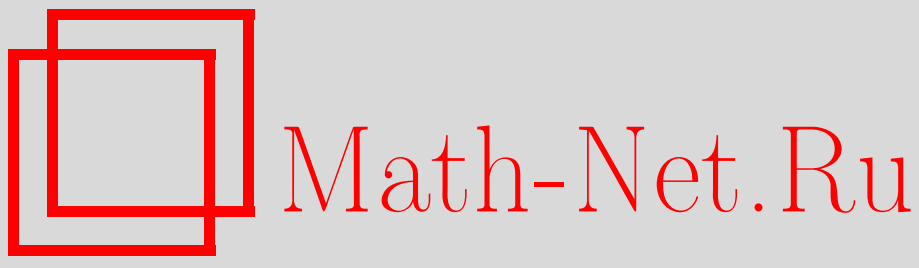

И. В. Камоцкий, С. А. Назаров, Аномалии Вуда и поверхностные волны в задаче рассеяния на периодической границе. II, Матем. сб., 1999, том 190, номер 2, 43-70

DOI: https://doi.org/10.4213/sm383

Использование Общероссийского математического портала Math-Net.Ru подразумевает, что вы прочитали и согласны с пользовательским соглашением http://www . mathnet.ru/rus/agreement

Параметры загрузки:

IP : 54.205 .225 .156

26 апреля 2023 г., $12: 55: 20$ 


\title{
Аномалии Вуда и поверхностные волны в задаче рассеяния на периодической границе. II
}

\begin{abstract}
Исследуется решение задачи дифракции плоской акустической волны на периодической границе при значениях частоты, близких к пороговым. Показано, что при специальной геометрии периодической структуры упомянутые изменения дифракционной картины (аномалии Вуда) сопровождаются появлением поверхностных волн. Обоснование асимптотических формул (в частности и тех, которые были найдены в [1]) базируется на технике эквивалентных весовых норм в пространствах Соболева.

Библиографиия: 8 названий.
\end{abstract}

Настоящая статья служит непосредственньм продолжением работы авторов [1]. Помимо обоснования асимптотических представлений, полученных в [1], обсуждается новый аспект аномалий Вуда - возникновение поверхностных волн. Физическая сторона вопроса подробно описана во введении [1], относящемся к обеим частям работы. Для удобства нумерация параграфов и формул продолжает начатую в [1] (например, центральную теорему 2.17 о “считающем" свойстве расширенной матрицы рассеяния следует искать в первой части).

\section{§4. Поверхностные волны}

1. Ключевой трюк. В этом параграфе мы занимаемся построением поверхностных волн, т.е. элементов ядра оператора $\mathscr{A}(\beta)$ или, что то же, решений однородной задачи (2.4)-(2.6), экспоненциально затухающих на бесконечности. Поясним замысел, основанньй на использовании "считаюшего" свойства расширенной матрицы рассеяния $S$ (теорема 2.17). Упростим изложение, фиксировав $k_{0}^{2}=\alpha^{2}$ и превратив матрицы $S^{-}$и $\stackrel{\circ}{s}$ в скаляры (поскольку $T=1$ ). Рассматривается семейство задач в областях $\Pi(\mu)$, зависящих от малого параметра $\mu>0$ и таких, что $\Pi(0)=\dot{(}-\pi, \pi) \times(0,+\infty)$, т.е. $\Omega=\mathbb{R}_{+}^{2}$. Возмущение подбирается так, чтобы матрица рассеяния на пороге непрерьвно зависела от $\mu \in\left[0, \mu_{1}\right)$; обозначим через $S(\mu, \delta)$ и т.п. атрибуты задачи в $\Pi(\mu)$ в допороговой ситуации $k^{2}=k_{0}^{2}-\delta^{4}$. Пусть еше $\stackrel{\circ}{s}(\mu)$ - матрица рассеяния на пороге. В предельной $(\mu=0)$ задаче решения $\stackrel{\circ}{\eta}_{T}, Y_{T}^{-}$и матрицы $\stackrel{\circ}{s}(0)$ и $S(0, \delta)$ находятся явно: $S(0, \delta)=-i$ и $\stackrel{\circ}{s}(0)=1$ (критический случай). В то же время удается подобрать такое возмущение П $(\mu)$ полуполосы $\Pi(0)$, что $\stackrel{\circ}{s}(\mu) \neq 1$ при $\mu>0$, и, следовательно, согласно результатам п. $3 \S 3$

$$
S(\mu, \delta)=i+O(\delta)
$$

Работа выпо лнена при частичной финансовой поддержкепрограммы INTAS-RFBR (грант № 95-12). 
при любом фиксированном $\mu>0$.

Ясно, что матрица $S(\mu, \delta)$ зависит непрерьвно от $\mu$ при $\delta>0$. Кроме того, в силу (4.1) она доопределяется по непрерьвности: $S(\mu, 0)=i$ при $\mu>0$. Соединим точки $\left(\mu_{1}, 0\right)$ и $\left(0, \delta_{1}\right)$ путем внутри прямоугольника $\left(0, \mu_{1}\right] \times\left(0, \delta_{1}\right]$. Так как $S\left(\mu_{1}, 0\right)=i, S\left(0, \delta_{1}\right)=-i$ и $(1 \times 1)$-матрица $S(\mu, \delta)$ "живет" на единичной окружности, найдется точка $\left(\mu_{2}, \delta_{2}\right)$ пути, для которой $S\left(\mu_{2}, \delta_{2}\right)=-1$ или $S\left(\mu_{2}, \delta_{2}\right)=1$. Обе ситуации можно реализовать за счет выбора возмушения П $(\mu)$ полосы П $(0)$ (к примеру, удалением малых дисков из П $(0)$ и постановкой на их границах условий Дирихле или Неймана соответственно). Вспоминая формулу (2.40) ("считаюшее" свойство $S$ ), видим, что соотношение $S\left(\mu_{2}, \delta_{2}\right)=1$ гарантирует сушествование искомой поверхностной волны. При этом величину $k_{0}^{2}-\delta_{2}^{4}$ следует интерпретировать как изолированное собственное число оператора Лапласа, рассматриваемого в качестве неограниченного оператора в $L_{2}\left(\Pi\left(\mu_{2}\right)\right)$ с областью определения $D=\left\{u \in H^{2}\left(\Pi\left(\mu_{2}\right), \alpha\right): \partial_{n} u=0\right.$ на $\left.\Gamma\left(\mu_{2}\right)\right\}$. В следующих разделах мы реализуем намеченный здесь план в более сложной ситуации и, в частности, обнаружим для оператора Лапласа с областью определения $D$ точечный спектр на непрерывном спектре $\left[\alpha^{2},+\infty\right)$.

2. Сингулярно возмушенная задача. Пусть П $(0)$ - полуполоса $(-\pi, \pi) \times$ $(0, \infty)$; при этом $\Gamma(0)=(-\pi, \pi) \times\{0\}$. Внутри П $(0)$ фиксируем две точки $x^{1}=$ $\left(x_{1}^{1}, x_{2}^{1}\right)$ и $x^{2}=\left(x_{1}^{2}, x_{2}^{2}\right)$, а затем вырежем из П $(0)$ два круга $D_{\varepsilon}^{1}$ и $D_{\varepsilon}^{2}$ с радиусами $\varepsilon$ и центрами в этих точках. Полученную область обозначим через П $(\varepsilon)$,

$$
\begin{aligned}
\Pi(\varepsilon) & =\Pi(0) \backslash\left(D_{\varepsilon}^{1} \cup D_{\varepsilon}^{2}\right), \\
\Gamma(\varepsilon) & =\Gamma(0) \cup \partial D_{\varepsilon}^{1} \cup \partial D_{\varepsilon}^{2} .
\end{aligned}
$$

Отметим, что в обозначениях из п. 1 параметр $\mu$ берется равным $\varepsilon$. Введем полярные координаты $\left(r_{i}, \varphi_{i}\right)$ с центрами в $x^{i}: r_{i}=\left|x-x^{i}\right|, \varphi \in[0,2 \pi)$.

Пусть, как и прежде, вьполнено соотношение (1.4), т.е. $0>\alpha>-1 / 2$. Положим $k_{0}^{2}=(1+\alpha)^{2}$; тогда на вещественной оси расположено четыре собственных числа задачи (2.1) при $k^{2}=k_{0}^{2}$. Введем оператор

$$
L_{\delta}(\nabla)=-\left(\Delta+k_{0}^{2}-\delta^{4}\right) .
$$

Как уже пояснялось, основная цель данного параграфа - показать, что при некоторых $x^{i}$ и $\varepsilon>0, \lambda \in\left[\frac{1}{2} k_{0}^{2}, 2 k_{0}^{2}\right]$ существует нетривиальное экспоненциально затухающее на бесконечности решение $u^{\varepsilon}$ уравнений

$$
\begin{aligned}
& L_{\lambda \varepsilon}(\nabla) u^{\varepsilon}(x)=0, \quad x \in \Pi(\varepsilon), \\
& \partial_{n} u^{\varepsilon}(x)=0, \quad x \in \Gamma(\varepsilon),
\end{aligned}
$$

дополненных условиями квазипериодичности. 
Зафиксируем весовой показатель $\beta>0$ так, чтобы в полосе $\{z \in \mathbb{C}:|\operatorname{Im} z|<\beta\}$ содержались только вещественные собственные числа задачи (2.1) при $k^{2}=k_{0}^{2}$. Найдется такое $\varepsilon_{0}>0$, что для $\varepsilon \in\left(0, \varepsilon_{0}\right)$ и $k^{2}=k_{0}^{2}-\lambda^{4} \varepsilon^{4}$ задача $(2.1)$ имеет в точности четыре собственных числа внутри той же полосы. Как и в $\S 2$, определим две приходящие и две уходящие волны; именно, в случае $\varepsilon=0$

$$
\begin{aligned}
& \stackrel{\circ}{u_{1}^{ \pm}}=\frac{1}{2}\left(\pi^{2}(1+2 \alpha)\right)^{-1 / 4} \exp \left[i\left(\alpha x_{1} \mp(1+2 \alpha)^{1 / 2} x_{2}\right)\right], \\
& \stackrel{\circ}{u_{2}^{ \pm}}=(4 \pi)^{-1 / 2} \exp \left[i(\alpha+1) x_{1}\right]\left(1 \mp i x_{2}\right),
\end{aligned}
$$

а если $\varepsilon>0$, то

$$
\begin{aligned}
& u_{1}^{ \pm}=\left(4 \pi\left(1+2 \alpha-\lambda^{4} \varepsilon^{4}\right)^{1 / 2}\right)^{-1 / 2} \exp \left[i\left(\alpha x_{1} \mp\left(1+2 \alpha-\lambda^{4} \varepsilon^{4}\right)^{1 / 2} x_{2}\right)\right] \\
& u_{2}^{ \pm}=(8 \pi)^{-1 / 2}(\lambda \varepsilon)^{-1} \exp \left[i(\alpha+1) x_{1}\right]\left(\exp \left[-\varepsilon^{2} \lambda^{2} x_{2}\right] \mp i \exp \left[\varepsilon^{2} \lambda^{2} x_{2}\right]\right) .
\end{aligned}
$$

В согласии с теоремой 2.11 мы получаем расширенную матрицу рассеяния размером $2 \times 2$. В силу теоремы 2.17 достаточно найти $\lambda, x^{i}$ и $\varepsilon>0$, для которых $S_{2,2}=1$. Поэтому далее мы оперируем только с нижней строкой матрицы $S$. Эта строка восстанавливается по квазипериодическому решению $Y_{2}$ задачи

$$
\begin{aligned}
& L_{\lambda \varepsilon}(\nabla) Y_{2}(x)=0, \quad x \in \Pi(\varepsilon), \\
& \partial_{n} Y_{2}(x)=0, \quad x \in \Gamma(\varepsilon),
\end{aligned}
$$

допускающему представление

$$
Y_{2}=u_{2}^{+}+S_{2,1} u_{1}^{-}+S_{2,2} u_{2}^{-}+Y_{2}^{\prime}
$$

с остатком $Y_{2}^{\prime} \in W_{\beta}^{l+1}(\Pi(\varepsilon) ; \alpha)$. Это решение $Y_{2}$, как и прежде, ишется в пространстве $W_{-\beta}^{l+1}(\Pi(\varepsilon) ; \alpha)$. Однако для нужд асимптотического анализа мы снабдим это пространство эквивалентной нормой, которая вводится при помощи разбиения единицы и выявляет зависимость решения от малого параметра. Так, если носитель функции не пересекается с дисками $D_{\delta}^{1}$ и $D_{\delta}^{2}$, где $\delta>0$ - фиксированное малое число, то ее новая норма не отличается от старой. В случае $\operatorname{supp} u \subset D_{\delta}^{j}$ эта норма совпадает с

$$
\left(\sum_{|\tau| \leqslant l+1} \int_{D_{\delta}^{j} \backslash D_{\varepsilon}^{j}} r_{j}^{2(\gamma-l-1+|\tau|)}\left|\nabla^{\tau} u(x)\right|^{2} d x\right)^{1 / 2}
$$

здесь $\gamma \in \mathbb{R}$ - дополнительный весовой показатель. При этом (4.12) - обычная норма в пространствах Кондратьева [2] (см. также [3; гл. 4]); она однородна относительно растяжений координат и наилучшим образом приспособлена к отслеживанию асимптотических зависимостей от $\varepsilon$. Пространство $W_{-\beta}^{l+1}(\Pi(\varepsilon) ; \alpha)$, 
снабженное указанной нормой, обозначим через $\mathscr{V}_{-\beta, \gamma}^{l+1}(\Pi(\varepsilon) ; \alpha)$. Нетрудно убедиться в том, что при любых $\beta, \gamma, \lambda \in\left[\frac{1}{2} k_{0}^{2}, 2 k_{0}^{2}\right]$ и $l=1,2, \ldots$ норма оператоpa $L_{\varepsilon}(\nabla): \mathscr{V}_{-\beta, \gamma}^{l+1}(\Pi(\varepsilon) ; \alpha) \rightarrow \mathcal{V}_{-\beta, \gamma}^{l-1}(\Pi(\varepsilon) ; \alpha)$ ограничена константой, не зависящей от $\lambda$ и $\varepsilon$. Пространство следов на $\Gamma(\varepsilon)$ нормальных производных функций из $\mathscr{V}_{-\beta, \gamma}^{l+1}(\Pi(\varepsilon) ; \alpha)$ обозначаем через $\mathcal{V}_{-\beta, \gamma}^{l-1 / 2}(\Gamma(\varepsilon) ; \alpha)$ и наделяем естественной нормой

$$
\left\|v ; \mathscr{V}_{-\beta, \gamma}^{l-1 / 2}(\Gamma(\varepsilon) ; \alpha)\right\|=\inf \left\{\left\|u ; \mathscr{V}_{-\beta, \gamma}^{l+1}(\Pi(\varepsilon) ; \alpha)\right\|: \partial_{n} u=v \text { на } \Gamma(\varepsilon)\right\} .
$$

Разумеется, она не зависит от выбора весового показателя $\beta$, обслуживающего бесконечность. Если $\operatorname{supp} v \subset \Gamma(0)$, то эта норма эквивалентна норме в $W_{-\beta}^{l-1 / 2}(\Gamma(0))$. Можно проверить, что в случае $\operatorname{supp} v \subset \partial D_{\varepsilon}^{j}$ эквивалентная норма выглядит так:

$$
\left(\varepsilon^{2(\gamma-l+1 / 2)}\left\|v ; L_{2}\left(\partial D_{\varepsilon}^{j}\right)\right\|^{2}+\varepsilon^{2 \gamma}\left\|v ; W_{-\beta}^{l-1 / 2}\left(\partial D_{\varepsilon}^{j}\right)\right\|^{2}\right)^{1 / 2} .
$$

3. Построение приближенного решения. Займемся построением приближенного решения задачи (4.9)-(4.11), (2.6). Как и в п. $3 \S 3$, около бесконечности (при $x_{2}>2 N|\ln \varepsilon|$ с некоторым положительным $N$ ) это решение представим как линейную комбинацию

$$
U^{\mathrm{I}}=u_{2}^{+}+S_{1}^{\prime} u_{1}^{-}+S_{2}^{\prime} u_{2}^{-},
$$

коэффициенты которой имеют вид

$$
\begin{gathered}
S_{1}^{\prime}=S_{1}^{(0)}+\varepsilon S_{1}^{(1)}+\varepsilon^{2} S_{1}^{(2)} \\
S_{2}^{\prime}=S_{2}^{(0)}+\varepsilon S_{2}^{(1)} .
\end{gathered}
$$

В соответствии с методом сращиваемых разложений (см. [4]) на усеченной "полуполосе" $\left\{x \in \Pi(\varepsilon): x_{2}<2 N|\ln \varepsilon|\right\}$ используется иной анзац, который согласуется с (4.15). Эта полуполоса сингулярно возмущена малыми отверстиями $D_{\varepsilon}^{j}$, $j=1,2, \ldots$, т.е., вообще говоря, необходимо ввести дополнительные анзацы, описывающие поведение решения в непосредственной близости от $D_{\varepsilon}^{j}$. Однако благодаря простой геометрической структуре возмушения можно обойтись и без них, а приближенное решение попросту искать в виде

$$
U^{\mathrm{II}}=\sum_{j=-1}^{2} \varepsilon^{j} V_{j}(x)+\widetilde{U}^{\mathrm{II}}, \quad x \in\left\{\Pi(\varepsilon): x_{2}<2 N|\ln \varepsilon|\right\} .
$$

Здесь $\widetilde{U}^{\mathrm{II}}$ - поправочный член, отличный от нуля только в окрестностях точек $x^{1}$ и $x^{2}$, а слагаемые $V_{j}$ являются квазипериодическими решениями задач

$$
\begin{aligned}
-\left(\Delta+k_{0}^{2}\right) V_{j}(x) & =0, \quad x \in \Pi(0) \backslash\left\{x^{1}, x^{2}\right\}, \\
\frac{\partial}{\partial x_{2}} V_{j}(x) & =0, \quad x \in \Gamma(0),
\end{aligned}
$$


причем их рост на бесконечности ограничивается включением в класс

$$
W_{-\beta}^{l+1}\left(\Pi(0) \backslash\left\{x: x_{2}<N\right\} ; \alpha\right)
$$

но им разрешается обладать сингулярностями в точках $x^{i}$. Очевидно, что $V_{j}$ не определяется однозначно, и тому имеются две причины. Во-первых, ядро оператора $\mathscr{A}(-\beta)$ нетривиально, т.е.

$$
V_{j}=\widetilde{V}_{j}+a_{1}^{(j)} \stackrel{\circ}{\eta}_{1}+a_{2}^{(j)} \stackrel{\circ}{\eta}_{2}
$$

где $\widetilde{V}_{j}$ - частное решение, а $a_{i}^{(j)}$-произвольные постоянные. Во-вторых, однородное уравнение (4.17) не обязано выполняться в точках $x^{1}$ и $x^{2}$, а значит, в его правую часть можно поместить линейную комбинацию $\delta$-функций Дирака и их производных, сосредоточенных в $x^{i}$. Для описания возможных сингулярностей $V_{j}$ введем функцию Грина $G(x, y)$ задачи $(4.17),(4.18)$ с условиями квазипериодичности и естественньми условиями излучения (см. [5]). Сушествование функции Грина вытекает из теоремы 2.16, гарантируюшей однозначную разрешимость такой задачи. При $j=1,2$ положим

$$
G_{j 0}(x)=\left.G(x, y)\right|_{y=x^{j}}, \quad G_{j 1}(x)=\left.\frac{\partial}{\partial y_{1}} G(x, y)\right|_{y=x^{j}}
$$

Справедливы представления

$$
\begin{array}{lll}
G_{j 0}(x)=-(2 \pi)^{-1} \ln r_{j}+G_{j 0}^{0, j}+O\left(r_{j}^{1-\delta}\right) & \text { при } r_{j} \rightarrow 0, \\
G_{j 1}(x)=(2 \pi)^{-1} r_{j}^{-1} \cos \varphi_{j}+G_{j 1}^{0, j}+O\left(r_{j}^{1-\delta}\right) & \text { при } r_{j} \rightarrow 0 .
\end{array}
$$

Здесь $\delta$ - любое положительное число. Соотношения (4.20), (4.21) можно почленно дифференцировать, приняв следуюшее соглашение:

$$
\nabla O\left(r_{j}^{\Lambda}\right)=O\left(r_{j}^{\Lambda-1}\right)
$$

Ясно, что для $G_{j 0}$ и $G_{j 1}$ справедливы равенства $(4.17),(4.18)$ и включение в класс (4.19). Обсудим подробнее поведение $G_{j m}$ при $x_{2} \rightarrow+\infty$. Так как $G_{j m}$ удовлетворяют условиям излучения, то

$$
G_{j m}=p_{j m}^{(1)} \stackrel{\circ}{u_{1}^{-}}+p_{j m}^{(2)} \stackrel{\circ}{u}_{2}^{-}+\cdots
$$

где многоточием обозначены элементы из $W_{\beta}^{l+1}\left(\Pi(0) \backslash\left\{x_{2}>R\right\} ; \alpha\right)$. Вычислим коэффициенты $p_{j m}^{(n)}$ при $j, n=1,2$ и $m=0,1$. С этой целью рассмотрим область $\Pi^{R} \backslash D_{\gamma}^{j}-$ прямоугольник $(-\pi, \pi) \times(0, R)$, из которого вырезан диск с малым радиусом $\gamma$ и центром $x^{j}$. На этой области справедливо равенство

$$
L_{0}(\nabla) G_{j 0}=0
$$


У множим (4.23) скалярно на $\stackrel{\circ}{\eta}_{m}$ и, применив несколько раз формулу Грина, получим:

$$
\begin{aligned}
& 0=\left(L_{0}(\nabla) G_{j 0}, \stackrel{\circ}{\eta}\right)_{\Pi^{R} \backslash D_{\gamma}^{j}}=-\left(\partial_{n} G_{j 0}, \stackrel{\circ}{\eta}_{m}\right)_{\partial\left(\Pi^{R} \backslash D_{\gamma}^{j}\right)}+\left(G_{j 0}, \partial_{n} \stackrel{\circ}{\eta}_{m}\right)_{\partial\left(\Pi^{R} \backslash D_{\gamma}^{j}\right)} \\
& -\left(\partial_{x_{2}}\left(p_{j 0}^{(1)} \stackrel{\circ}{u_{1}^{-}}+p_{j 0}^{(2)} \stackrel{\circ}{u_{2}^{-}}+\cdots\right), \stackrel{\circ}{u}_{m}^{+}+\stackrel{\circ}{u}_{m}^{-}\right)_{\left\{x_{2}=R,\left|x_{1}\right|<\pi\right\}} \\
& +\left(p_{j 0}^{(1) \stackrel{\circ}{u}}-p_{1}^{-}+p_{j 0}^{(2)} \stackrel{\circ}{u_{2}^{-}}+\cdots, \partial_{x_{2}}\left(\stackrel{\circ}{u}_{m}^{+}+\stackrel{\circ}{u}_{m}^{-}\right)\right)_{\left\{x_{2}=R,\left|x_{1}\right|<\pi\right\}} \\
& +\left(\frac{\partial}{\partial r_{j}}\left(-(2 \pi)^{-1} \ln r_{j}+O(1)\right), \stackrel{\circ}{\eta}_{m}\left(x^{j}\right)+O\left(r_{j}\right)\right)_{r_{j}=\gamma} \\
& -\left(-(2 \pi)^{-1} \ln r_{j}+O(1), \frac{\partial}{\partial r_{j}}\left(\stackrel{\circ}{\eta}_{m}\left(x^{j}\right)+O\left(r_{j}\right)\right)\right) .
\end{aligned}
$$

Ввиду экспоненциального затухания при $x_{2} \rightarrow \infty$ вклад остатков “. . ." в интегралы по отрезкам $\left\{x:\left|x_{1}\right|<\pi, x_{2}=R\right\}$ оказывается бесконечно мальм при $R \rightarrow \infty$. Интегралы по дуге $\left\{x: r_{j}=\gamma\right\}$, содержащие символы $O(1)$ и $O\left(r_{j}\right)$, также исчезают при $\gamma \rightarrow 0$. Выполним указанные предельные переходы, и формула (4.24)

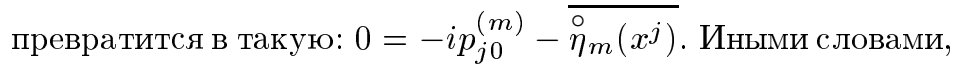

$$
p_{j 0}^{(m)}=i \overline{\stackrel{\supset}{\eta}_{m}\left(x^{j}\right)}, \quad j, m=1,2 .
$$

Аналогичные рассуждения применительно к $G_{j 1}$ приводят к равенствам

$$
p_{j 1}^{(m)}=i \overline{\frac{\partial}{\partial x_{1}} \stackrel{\circ}{\eta}_{m}\left(x^{j}\right)}, \quad j, m=1,2 .
$$

Выпишем асимптотические разложения, которые будут востребованы далее. Для $G_{j m}$ имеем

$$
\begin{aligned}
G_{j 0}= & -(2 \pi)^{-1} \delta_{j n} \ln r_{n}+G_{j 0}^{0, n}+\sum_{p=1}^{2} G_{j 0}^{p, n}\left(x_{p}-x_{p}^{n}\right)+O\left(r_{n}^{2-\delta}\right), r_{n} \rightarrow 0, \\
G_{j 1}= & (2 \pi)^{-1} \delta_{j n} r_{n}^{-1} \cos \varphi_{n}+G_{j 1}^{0, n}+\sum_{p=1}^{2} G_{j 1}^{p, n}\left(x_{p}-x_{p}^{n}\right) \\
& -k_{0}^{2}(4 \pi)^{-1} \delta_{j n} r_{n} \ln r_{n} \cos \varphi_{n}+O\left(r_{n}^{2-\delta}\right), \quad r_{n} \rightarrow 0,
\end{aligned}
$$

Кроме того,

$$
\begin{aligned}
\frac{\partial}{\partial r_{n}} G_{j 0}= & -(2 \pi)^{-1} \delta_{j n} r_{n}^{-1}+G_{j 0}^{1, n} \cos \varphi_{n}+G_{j 0}^{2, n} \sin \varphi_{n}+O\left(r_{n}^{1-\delta}\right), \quad r_{n} \rightarrow 0 \\
\frac{\partial}{\partial r_{n}} G_{j 1}= & -(2 \pi)^{-1} \delta_{j n} r_{n}^{-2} \cos \varphi_{n}+G_{j 1}^{1, n} \cos \varphi_{n}+G_{j 1}^{2, n} \sin \varphi_{n} \\
& -k_{0}^{2}(4 \pi)^{-1} \delta_{j n}\left(\ln r_{n}+1\right) \cos \varphi_{n}+O\left(r_{n}^{2-\delta}\right), \quad r_{n} \rightarrow 0
\end{aligned}
$$


Так как $\stackrel{\circ}{\eta}_{2}$ зависит только от $x_{2}-x_{2}^{n}=r_{n} \cos \varphi_{n}$, получаем

$$
\begin{aligned}
& \stackrel{\circ}{\eta}_{2}(x)=\sum_{p=0}^{3} C_{n}^{(p)} r_{n}^{p} \cos ^{p} \varphi_{n}+O\left(r_{n}^{4}\right), \quad r_{n} \rightarrow 0, \\
& \frac{\partial}{\partial r_{n}} \stackrel{\circ}{\eta}_{2}(x)=\sum_{p=1}^{3} C_{n}^{(p)} p r_{n}^{p-1} \cos ^{p} \varphi_{n}+O\left(r_{n}^{3}\right), \quad r_{n} \rightarrow 0 .
\end{aligned}
$$

Здесь индексы $j, n$ принимают значения 1,2 , через $G_{\ldots}^{\ldots}$ обозначены какие-либо постоянные,

$$
C_{n}^{(p)}=\left.\frac{1}{p !}\left(\frac{\partial^{p}}{\partial x_{1}^{p}} \stackrel{\circ}{2}_{2}\right)\right|_{r_{n}=0}, \quad p=0,1,2,3, \quad n=1,2
$$

ЗАмечАниЕ 4.1 . Норма $\left\|\cdot ; \mathscr{V}_{\beta, l}^{l-1 / 2}\left(S_{\varepsilon}^{n}\right)\right\|$ остатков в $(4.29),(4.30)$ ввиду $(4.14)$ не превосходят $C \varepsilon^{2-\delta}$. Из (4.14) также следует, что норма $\left\|\cdot ; \mathscr{V}_{\beta, l}^{l-1 / 2}\left(S_{\varepsilon}^{n}\right)\right\|$ остатка в $(4.32)$ меньше чем $C \varepsilon^{4}$.

Положим

$$
\begin{aligned}
V_{m} & =a^{(m)} \stackrel{\circ}{\eta}_{2}, & m & =-1,0 \\
V_{m} & =\sum_{j=1}^{2} \sum_{n=0}^{1} b_{j, n}^{(m)} G_{j n}+a^{(m)} \stackrel{\circ}{\eta}_{2}, & m & =1,2 .
\end{aligned}
$$

Ясно, что сумма $\sum_{m=-1}^{2} \varepsilon^{m} V_{m}$ порождает малую невязку в уравнении (4.3). В то же время невязка в краевом условии (4.4) может оказаться недостаточно малой (для наших целей). Покажем, как можно устранить это препятствие с помощью поправочного члена $\widetilde{U}^{\mathrm{II}}$. Имеем

$$
\begin{aligned}
& \left.\frac{\partial}{\partial r_{n}} \sum_{m=-1}^{2} \varepsilon^{m} V_{m}\right|_{r_{n}=\varepsilon} \\
& \quad=\sum_{m=-1}^{1} \varepsilon^{m} a^{(m)} \sum_{p=1}^{2-m} C_{n}^{(p)} p \varepsilon^{p-1} \cos ^{p} \varphi_{n}+\varepsilon(-2 \pi)^{-1}\left(\varepsilon^{-1} b_{n, 0}^{(1)}+\varepsilon^{-2} b_{n, 1}^{(1)} \cos \varphi_{n}\right) \\
& \quad+\varepsilon\left\{\sum_{j=1}^{2} \sum_{p=0}^{1} b_{j, p}^{(1)}\left(G_{j p}^{1, n} \cos \varphi_{n}+G_{j n}^{2, n} \sin \varphi_{n}\right)-k_{0}^{2}(4 \pi)^{-1} b_{n, 1}^{(1)}(\ln \varepsilon+1) \cos \varphi_{n}\right\} \\
& \quad+\varepsilon^{2}(-2 \pi)^{-1}\left(\varepsilon^{-1} b_{n, 0}^{(2)}+\varepsilon^{-2} b_{n, 1}^{(2)} \cos \varphi_{n}\right)+O\left(\varepsilon^{2-\delta}\right) .
\end{aligned}
$$


Группируя выражения с одинаковьми степенями $\varepsilon$, получаем

$$
\begin{aligned}
& \left.\frac{\partial}{\partial r_{j}} \sum_{m=-1}^{2} \varepsilon^{m} V_{m}\right|_{r_{j}=\varepsilon} \\
& =\sum_{m=-1}^{1} \varepsilon^{m} \sum_{n=1}^{m+2} C_{j}^{(n)} n a^{(m-n+1)} \cos ^{n} \varphi_{j}+\varepsilon^{-1}(-2 \pi)^{-1} b_{j, 1}^{(1)} \cos \varphi_{j} \\
& +\varepsilon^{0}(-2 \pi)^{-1}\left(b_{j, 0}^{(1)}+b_{j, 1}^{(2)} \cos \varphi_{j}\right) \\
& +\varepsilon\left\{b_{j, 0}^{(2)}(-2 \pi)^{-1}+\sum_{n=1}^{2} \sum_{p=0}^{1} b_{n, p}^{(1)}\left(G_{n p}^{1, j} \cos \varphi_{j}\right.\right. \\
& \left.\left.+G_{n p}^{2, j} \sin \varphi_{j}\right)+k_{0}^{2}(-4 \pi)^{-1} b_{j, 1}^{(1)}(\ln \varepsilon+1) \cos \varphi_{j}\right\}+O\left(\varepsilon^{2-\delta}\right) \\
& =\varepsilon^{-1}\left\{a^{(-1)} C_{j}^{(1)}-(2 \pi)^{-1} b_{j, 1}^{(1)}\right\} \cos \varphi_{j}+\varepsilon^{0}\left(\left\{a^{(-1)} C_{j}^{(2)}-(2 \pi)^{-1} b_{j, 0}^{(1)}\right\}\right. \\
& \left.+a^{(-1)} C_{j}^{(2)} \cos 2 \varphi_{j}+\left\{a^{(0)} C_{j}^{(1)}-(2 \pi)^{-1} b_{j, 1}^{(2)}\right\} \cos \varphi_{j}\right) \\
& +\varepsilon\left(\left\{a^{(0)} C_{j}^{(2)}-(2 \pi)^{-1} b_{j, 0}^{(2)}\right\}+3 a^{(-1)} C_{j}^{(3)} \frac{3 \cos \varphi_{j}+\cos 3 \varphi_{j}}{4}\right. \\
& +\sum_{n=1}^{2} \sum_{p=0}^{1} b_{n, p}^{(1)}\left(G_{n p}^{1, j} \cos \varphi_{j}+G_{n p}^{2, j} \sin \varphi_{j}\right)+a^{(0)} \cos 2 \varphi_{j} C_{j}^{(2)} \\
& \left.+a^{(1)} C_{j}^{(1)} \cos \varphi_{j}-k_{0}^{2}(4 \pi)^{-1} b_{j, 1}^{(1)}(\ln \varepsilon+1) \cos \varphi_{j}\right)+O\left(\varepsilon^{2-\delta}\right) .
\end{aligned}
$$

Положив

$$
b_{n, p}^{(m)}=2 \pi a^{(m-2)} C_{n}^{(2-p)}, \quad n, m=1,2, \quad p=0,1
$$

уничтожим часть слагаемых в $(4.35)$ и получим

$$
\begin{aligned}
& \left.\frac{\partial}{\partial r_{j}} \sum_{m=-1}^{2} \varepsilon^{m} V_{m}\right|_{r_{j}=\varepsilon}=a^{(-1)} C_{j}^{(2)} \cos 2 \varphi_{j}+\varepsilon\left(3 a^{(-1)} C_{j}^{(3)} \frac{3 \cos \varphi_{j}+\cos 3 \varphi_{j}}{4}\right. \\
& \quad+\sum_{n=1}^{2} \sum_{p=0}^{1} b_{n, p}^{(1)}\left(G_{n p}^{1, j} \cos \varphi_{j}+G_{n p}^{2, j} \sin \varphi_{j}\right)+a^{(0)} C_{j}^{(2)} \cos 2 \varphi_{j} \\
& \left.\quad+a^{(1)} C_{j}^{(1)} \cos \varphi_{j}+k_{0}^{2}(-4 \pi)^{-1} b_{j, 1}^{(1)}(\ln \varepsilon+1) \cos \varphi_{j}\right)+O\left(\varepsilon^{2-\delta}\right) .
\end{aligned}
$$

Поскольку выбор коэффициентов $a^{(j)}$ не в нашей власти (они фиксируются при сращивании $U^{\mathrm{I}}$ и $\left.U^{\mathrm{II}}\right)$, выделенные в (4.37) члены невязки компенсируются попра- 
вочными членами. Положим

$$
\begin{gathered}
I_{3}=\sum_{j=1}^{2}\left(m_{3}^{j, 1} r_{j}^{-1} \sin \varphi_{j}+m_{3}^{j, 2} r_{j}^{-1} \cos \varphi_{j}+m_{3}^{j, 3} r_{j}^{-2} \cos 2 \varphi_{j}\right) \chi\left(r_{j} \delta_{1}^{-1}\right) \\
I_{4}=\sum_{j=1}^{2}\left(m_{4}^{j} r_{j}^{-2} \cos 2 \varphi_{j}\right) \chi\left(r_{j} \delta_{1}^{-1}\right), \\
I_{5}=\sum_{j=1}^{2}\left(m_{5}^{j} r_{j}^{-3} \cos 3 \varphi_{j}\right) \chi\left(r_{j} \delta_{1}^{-1}\right), \\
\widetilde{U}^{\mathrm{II}}=\sum_{j=3}^{5} \varepsilon^{j} I_{j}
\end{gathered}
$$

где $m_{\ldots} \ldots$ - произвольные константы. Теперь, вычисляя производную по $r_{j}$, находим

$$
\begin{aligned}
\left.\frac{\partial}{\partial r_{j}} \widetilde{U}^{\mathrm{II}}\right|_{r_{j}=\varepsilon}= & -\varepsilon\left(m_{3}^{j, 1} \sin \varphi_{j}+m_{3}^{j, 2} \cos \varphi_{j}\right. \\
& \left.+2 m_{4}^{j} \cos 2 \varphi_{j}+3 m_{5}^{j} \cos 3 \varphi_{j}\right)-2 m_{3}^{j, 3} \cos 2 \varphi_{j}
\end{aligned}
$$

Складывая (4.37), (4.38) и приравнивая к нулю коэффициенты при $\varepsilon^{j} \cos p \varphi_{n}$ и $\varepsilon^{j} \sin p \varphi_{n}$, выбираем $m \ldots$ так, чтобы

$$
\left.\frac{\partial}{\partial r_{j}} \widetilde{U}^{\mathrm{II}}\right|_{r_{j}=\varepsilon}=O\left(\varepsilon^{2-\delta}\right), \quad \varepsilon \rightarrow 0 .
$$

Рассмотрим поведение $U^{\mathrm{II}}$ около бесконечности. Имеем

$$
\begin{aligned}
U^{\mathrm{II}}= & \sum_{j=-1}^{2} \varepsilon^{j} V_{j}+\widetilde{U}^{\mathrm{II}}=\sum_{j=-1}^{0} \varepsilon^{j}\left(a^{(j)} \stackrel{\circ}{u_{2}^{+}}+a^{(j)} \stackrel{\circ}{u_{2}^{-}}\right) \\
& +\sum_{j=1}^{0} \varepsilon^{j}\left(a^{(j)}\left(\stackrel{\circ}{u}_{2}^{+}+\stackrel{\circ}{u_{2}^{-}}\right)+\sum_{n, d=1}^{2} \sum_{m=0}^{1} 2 \pi a^{(j-2)} C_{n}^{(2-m)} p_{n, m}^{(d)} \stackrel{\circ}{u}_{d}^{-}\right)+\cdots
\end{aligned}
$$

Обработаем величину $U^{\mathrm{I}}$; ее разложение в ряд по степеням $\varepsilon$ имеет вид

$$
U^{\mathrm{I}}=\sum_{j=-1}^{2} \varepsilon^{j} R_{j}+O\left(\varepsilon^{3} \ln ^{3} \varepsilon\right), \quad\left(x_{1}, x_{2}\right) \in \Omega_{\varepsilon}
$$

где

$$
\begin{aligned}
R_{-1} & =\lambda^{-1} \sigma_{+}\left(-i+S_{2}^{(0)}\right)\left(\stackrel{\circ}{u}_{2}^{+}+\stackrel{\circ}{u}_{2}^{-}\right), \\
R_{0} & =S_{1}^{(0)} \stackrel{\circ}{u}_{1}^{-}+\sigma_{+}\left(\stackrel{\circ}{u}_{2}^{+}+\stackrel{\circ}{u}_{2}^{-}\right) S_{2}^{(1)}, \\
R_{1} & =\lambda \sigma_{+}\left(i+S_{2}^{(0)}\right)\left(\stackrel{\circ}{u}_{2}^{-}-\stackrel{\circ}{u}_{2}^{+}\right)+S_{1}^{(1)} \stackrel{\circ}{u}_{1}^{-}, \\
R_{2} & =S_{1}^{(2)} \stackrel{\circ}{u}_{1}^{-}+\lambda^{2} \sigma_{+}\left(\stackrel{\circ}{u}_{2}^{-}-\stackrel{\circ}{u_{2}^{+}}\right) S_{2}^{(1)} .
\end{aligned}
$$


Вьполняя условие сращивания, приравниваем коэффициенты при $\varepsilon^{-1} \stackrel{\circ}{u} \underset{2}{ \pm}$ в (4.40) и в $(4.41)$ :

$$
a^{(-1)}=\lambda^{-1} \sigma_{+}\left(-i+S_{2}^{(0)}\right)
$$

Поступая аналогично с коэффициентами при $\varepsilon^{0} u_{j}^{ \pm}$, выводим формулы

$$
S_{1}^{(0)}=0, \quad S_{2}^{(1)} \sigma_{+}=a^{(0)}
$$

Сопоставляя множители при $\varepsilon \stackrel{\circ}{2}_{2}^{+}, \varepsilon \stackrel{\circ}{u}_{2}^{-}, \varepsilon \stackrel{\circ}{1}_{1}^{-}$, находим, что

$$
\begin{gathered}
a^{(1)}=-\lambda \sigma_{+}\left(i+S_{2}^{(0)}\right), \\
a^{(1)}+2 \pi \sum_{n=1}^{2} \sum_{m=0}^{1} a^{(-1)} C_{n}^{(2-m)} p_{n, m}^{(2)}=\lambda \sigma_{+}\left(i+S_{2}^{(0)}\right), \\
S_{1}^{(1)}=2 \pi a^{(-1)} \sum_{n=1}^{2} \sum_{m=0}^{1} C_{n}^{(2-m)} p_{n, m}^{(1)} .
\end{gathered}
$$

Равенства (4.44) и (4.45) влекут соотношение

$$
a^{(-1)} 2 \pi \sum_{n=1}^{2} \sum_{m=0}^{1} C_{n}^{(2-m)} p_{n, m}^{(2)}=2 \lambda \sigma_{+}\left(i+S_{2}^{(0)}\right) .
$$

Вспоминая формулы $(4.25),(4.26)$ и (4.33), получаем окончательно, что

$$
S_{2}^{(0)}=\frac{\lambda^{2}+i k_{0}^{2}}{k_{0}^{2}+i \lambda^{2}}
$$

Проводя аналогичные действия с коэффициентом при $\varepsilon^{2}$, выводим

$$
S_{1}^{(1)}=S_{1}^{(2)}=a^{(2)}=a^{(0)}=0
$$

Как и в п. $6 \S 3$, образуем приближенное решение задачи $(4.3),(4.4)$ в соответствии с формулой

$$
\Phi=U^{\mathrm{II}} \chi\left(x_{2}|N \ln \varepsilon|^{-1}\right)+\left(1-\chi\left(x_{2}|N \ln \varepsilon|^{-1}\right)\right) U^{\mathrm{I}} .
$$

Определенная таким образом функция $\Phi$ удовлетворят (4.3), (4.4). Покажем, что нормы невязок, порожденных $\Phi$ в соотношениях (4.3), (4.4), малы.

Из замечания 4.1 и формул (4.34), (4.39) вытекает неравенство

$$
\left\|\partial_{n} \Phi ; \mathscr{V}_{\beta, 1}^{l-1 / 2}(\Gamma(\varepsilon) ; \alpha)\right\| \leqslant C \varepsilon^{3-\gamma} .
$$


Помимо этого, из определения (4.50) следует, что функция $\Phi$ совпадает с $U^{\mathrm{I}}$ при $x_{2}>2 N|\ln \varepsilon|$. Значит,

$$
\begin{gathered}
L_{\lambda \varepsilon}(\nabla) \Phi(x)=0, \quad x \in \Pi(\varepsilon) \cap\left\{x_{2}>2 N|\ln \varepsilon|\right\}, \\
L_{\lambda \varepsilon}(\nabla) \Phi(x)=\lambda^{4} \varepsilon^{4} \sum_{j=-1}^{2} \varepsilon^{j} V_{j}(x)+\left(\lambda^{4} \varepsilon^{4}-k_{0}^{2}\right) \widetilde{U}^{\mathrm{II}}(x)+\Delta \widetilde{U}^{\mathrm{II}}(x), \\
x \in \Pi(\varepsilon) \cap\left\{x_{2}<2 N|\ln \varepsilon|\right\} .
\end{gathered}
$$

В соответствии с $(4.27),(4.28),(4.34)$ получаем

$$
\left\|\lambda^{4} \varepsilon^{4} \sum_{j=-1}^{2} \varepsilon^{j} V_{j}(x) ; \mathscr{V}_{\beta, l}^{l-1}\left(\Pi(\varepsilon) \cap\left\{x_{2}>2 N|\ln \varepsilon|\right\} ; \alpha\right)\right\| \leqslant C \varepsilon^{3-\gamma / 2-\beta N} ;
$$

здесь $\gamma$ - любое положительное число. Благодаря тому, что $\operatorname{supp} \Delta \widetilde{U}^{\mathrm{II}} \subset$ $\bigcup_{n=1}^{2} D_{2 \delta_{1}}^{n} \backslash D_{\delta_{1}}^{n}$, для третьего слагаемого в (4.53) выполнена оценка, аналогичная (4.54). В силу определения $J_{n}$ имеем

$$
\begin{aligned}
& \left\|J_{3} ; \mathscr{V}_{\beta, l}^{l-1}(\Pi(\varepsilon) ; \alpha)\right\| \leqslant C|\ln \varepsilon|^{1 / 2} \\
& \left\|J_{n} ; \mathcal{V}_{\beta, l}^{l-1}(\Pi(\varepsilon) ; \alpha)\right\| \leqslant C \varepsilon^{-1}, \quad n=4,5 .
\end{aligned}
$$

Таким образом, установлено неравенство

$$
\left\|L_{\lambda \varepsilon}(\nabla) \Phi ; \mathscr{V}_{\beta, l}^{l-1}\left(\Pi(\varepsilon) \cap\left\{x_{2}<N|\ln \varepsilon|\right\} ; \alpha\right)\right\| \leqslant C \varepsilon^{-\beta N+3-\gamma / 2} .
$$

Рассматривая $L_{\lambda \varepsilon}(\nabla) \Phi$ в области $\Omega_{\varepsilon}$ так же, как и в п. $6 \S 3$, получаем оценку, аналогичную (3.89),

$$
\left\|L_{\lambda \varepsilon}(\nabla) \Phi ; \mathscr{V}_{\beta, l}^{l-1}\left(\Omega_{\varepsilon} ; \alpha\right)\right\| \leqslant C|\ln \varepsilon| N\left(\varepsilon^{\left(\lambda_{*}-\beta\right) N-1}+\varepsilon^{3-2 \beta N}\right) .
$$

Выберем $\beta$ и $N$ так, чтобы оба слагаемых справа были не больше $C \varepsilon^{3-\gamma}$, т.е.

$$
\beta \leqslant \frac{\lambda_{*} \gamma}{8-\gamma}, \quad N=\frac{8-\gamma}{2 \lambda_{*}}
$$

Складывая (4.55), (4.56) и учитьвая (4.52), находим

$$
\left\|L_{\lambda \varepsilon}(\nabla) \Phi ; \mathcal{V}_{\beta, l}^{l-1}(\Pi(\varepsilon) ; \alpha)\right\| \leqslant C \varepsilon^{3-\gamma}
$$

(напомним, что $\gamma$ - любое положительное число).

В п. $7 \S 2$ было определено пространство $\widehat{\mathbb{W}}_{-} ;$снабдим его эквивалентной нормой $\left\|\cdot ; \mathbb{W}_{\beta, \gamma}^{l+1}(\Pi(\varepsilon) ; \alpha, \delta)\right\|$. Именно, для функций, допускающих представление

$$
u=\left(1-\chi\left(x_{2} R^{-1}\right)\right) \sum_{j=1}^{2} c_{j} u_{j}^{-}+u^{\prime}
$$


(остаток $u^{\prime}$ содержится в $W_{\beta}^{l+1}(\Pi(\varepsilon) ; \alpha), u_{j}^{-}$- уходящие волны, соответствуюшие $k^{2}=k_{0}^{2}-\delta^{4} ;$ напоминаем, что постоянная $\beta$ была выбрана так, что мы располагаем только двумя уходяшими волнами), положим

$$
\left\|u ; \mathbb{W}_{\beta, \gamma}^{l+1}(\Pi(\varepsilon) ; \alpha, \delta)\right\|=\left(\sum_{j=1}^{2}\left|c_{j}\right|^{2}+\left\|u^{\prime} ; \mathfrak{Y}_{\beta, \gamma}^{l+2}(\Pi(\varepsilon) ; \alpha)\right\|^{2}\right)^{1 / 2} .
$$

Здесь $R$ фиксировано условием: точки $x^{j}$, центры дисков, удовлетворяют неравенствам $x_{2}^{j}<R$. Из (4.11), (4.15) вытекает включение

$$
Y_{2}^{-}-\Phi \in \mathbb{W}_{\beta, l}^{l+1}(\Pi(\varepsilon) ; \alpha, \lambda \varepsilon),
$$

а представление (4.15), формулы (4.25), (4.26), (4.33), (4.46), (4.48) и (4.49) обеспечивают информацию о поведении $Y_{2}^{-}-\Phi$ при $x_{2} \rightarrow+\infty$ :

$$
\begin{aligned}
Y_{2}^{-}-\Phi= & \left\{S_{2,1}-\varepsilon \frac{i\left(\alpha^{2}-1\right) \lambda(1+i)}{(1+2 \alpha)^{1 / 4} \sqrt{2}\left(k_{0}^{2}+i \lambda^{2}\right)} \sum_{j=1}^{2} \exp \left(i x_{1}^{j}\right) \cos \left[(1+2 \alpha)^{1 / 2} x_{2}^{j}\right]\right\} u_{1}^{-} \\
& +\left\{S_{2,2}-\frac{\lambda^{2}+i k_{0}^{2}}{k_{0}^{2}+i \lambda^{2}}\right\} u_{2}^{-}+\cdots
\end{aligned}
$$

Оценки (4.51) и (4.58) влекут

$$
\begin{array}{r}
\left\|L_{(\lambda \varepsilon)}(\nabla)\left(Y_{2}^{-}-\Phi\right) ; \mathscr{V}_{\beta, l}^{l-1 / 2}(\Pi(\varepsilon) ; \alpha)\right\| \leqslant C \varepsilon^{3-\gamma}, \\
\left\|\partial_{n}\left(Y_{2}^{-}-\Phi\right) ; \mathscr{V}_{\beta, l}^{l-1 / 2}(\Gamma(\varepsilon) ; \alpha)\right\| \leqslant C \varepsilon^{3-\gamma} .
\end{array}
$$

ПреДЛОЖЕнИЕ 4.2. Существует такое $\varepsilon_{1}>0$, ито при $\varepsilon \in\left(0, \varepsilon_{1}\right)$ для решения $u \in \mathbb{W}_{\beta, l}^{l+1}(\Pi(\varepsilon) ; \alpha, \lambda \varepsilon)$ задачи

$$
\begin{array}{rlrl}
-\left(\Delta+k_{0}^{2}-(\lambda \varepsilon)^{4}\right) u & =f & \text { на } & \Pi(\varepsilon), \\
\partial_{n} u=g & \text { на } & \Gamma(\varepsilon),
\end{array}
$$

в которой $f \in \mathscr{V}_{\beta, l}^{l-1}(\Pi(\varepsilon) ; \alpha), g \in \mathcal{V}_{\beta, l}^{l-1 / 2}(\Gamma(\varepsilon) ; \alpha)$ u $\lambda \in\left[\frac{1}{2} k_{0}^{2}, 2 k_{0}^{2}\right]$, коэффициенты $c_{1}$ и с $c_{2}$ в его представлении

$$
u=c_{1} u_{1}^{-}+c_{2} u_{2}^{-}+\cdots
$$

подчинены неравенству

$$
\left|c_{1}\right|+\left|c_{2}\right| \leqslant C_{\gamma} \varepsilon^{-1-\gamma}\left\|\{f, g\} ; \mathscr{V}_{\beta, l}^{l-1}(\Pi(\mu) ; \alpha) \times \mathcal{V}_{\beta, l}^{l-1 / 2}(\Gamma(\mu) ; \alpha)\right\|,
$$

әде $\gamma \in(0,1)$, а константа $C_{\gamma}$ не зависит от $\varepsilon, \lambda u x^{1}, x^{2}$.

Доказательство этого утверждения вынесено в отдельный параграф (см. замечание 5.8) по причине его независимости от излагаемого сейчас материала. Из предложения 4.2 и оценок (4.60), (4.61) вытекает, что для коэффициентов при уходящих волнах в (4.60) верны соотношения

$$
\begin{gathered}
\left|S_{2,2}-\frac{\lambda^{2}+i k_{0}^{2}}{k_{0}^{2}+i \lambda^{2}}\right| \leqslant C \varepsilon^{2(1-\gamma)} \\
\left|S_{1,2}-\varepsilon \frac{i\left(\alpha^{2}-1\right) \lambda(1+i)}{(1+2 \alpha)^{1 / 4} \sqrt{2}\left(k_{0}^{2}+i \lambda^{2}\right)} \sum_{j=1}^{2} \exp \left(i x_{1}^{j}\right)\left[\cos (1+2 \alpha)^{1 / 2} x_{2}^{j}\right]\right| \leqslant C \varepsilon^{2(1-\gamma)} .
\end{gathered}
$$

Итак, нами получены асимптотические приближения элементов $S_{2,1}$ и $S_{2,2}$ нижней строчки расширенной матрицы рассеяния. 
4. Анализ асимптотических формул. Цель этого пункта - показать, что за счет выбора параметров $\varepsilon, \lambda, x^{1}, x^{2}$ можно добиться равенства

$$
S_{2,2}=1
$$

Для этого естественно воспользоваться формулами (4.65), (4.66). Кроме того, в силу соотношения

$$
\left|S_{2,1}\right|^{2}+\left|S_{2,2}\right|^{2}=1
$$

достаточно проверить, что при должном выборе параметров достигаются равенства

$$
\begin{aligned}
& 0=\operatorname{Im} S_{2,2}, \\
& 0=S_{2,1} .
\end{aligned}
$$

Заметим, что соотношение (4.68) следует из унитарности расширенной матрицы рассеяния (см. теорему 2.11) и оценки (4.65), показывающей, что при достаточно малых $\varepsilon, \lambda \in\left[\frac{1}{2} k_{0}^{2}, 2 k_{0}^{2}\right]$ выполнено неравенство

$$
\operatorname{Re} S_{2,2}>0
$$

Уравнение (4.69) согласно оценке (4.65) может быть переписано в виде

$$
0=\frac{k_{0}^{4}-\lambda^{4}}{k_{0}^{4}+\lambda^{4}}+S_{2,2}^{\prime}
$$

причем для остатка получаем

$$
\left|S_{2,2}^{\prime}\right| \leqslant C \varepsilon^{3 / 2} .
$$

Точно так же из (4.66) и (4.70) вытекает соотношение

$$
0=\varepsilon \frac{i\left(\alpha^{2}-1\right) \lambda(1+i)}{(1+2 \alpha)^{1 / 4} \sqrt{2}\left(k_{0}^{2}+i \lambda^{2}\right)} \sum_{j=1}^{2} \exp \left(i x_{1}^{j}\right) \cos \left[(1+2 \alpha)^{1 / 2} x_{2}^{j}\right]+S_{2,1}^{\prime}
$$

здесь для $S_{2,1}^{\prime}$ выполнена оценка, подобная (4.72). Так как нас интересует решение уравнений при $\varepsilon>0$ и поскольку выражение в фигурных скобках в (4.73) не равно нулю при $\lambda \in\left[\frac{1}{2} k_{0}^{2}, 2 k_{0}^{2}\right]$, формула (4.73) может быть переписана так:

$$
0=\sum_{j=1}^{2} \exp \left(i x_{1}^{j}\right) \cos \left[(1+2 \alpha)^{1 / 2} x_{2}^{j}\right]+S_{2,1}^{\prime \prime}
$$

При этом

$$
\left|S_{2,2}^{\prime \prime}\right| \leqslant C \varepsilon^{1 / 2} .
$$

Наконец, (4.71) преобразуется в равенство

$$
0=\left(k_{0}^{4}-\lambda^{4}\right)+S_{2,2}^{\prime \prime}
$$


с аналогичной (4.75) оценкой для $S_{2,2}^{\prime \prime}$.

Положим $x_{1}^{1}=0, x_{1}^{2}=\pi / 2$; тогда из (4.74) вытекают два вещественных уравнения

$$
\begin{aligned}
& 0=\cos \left[(1+2 \alpha)^{1 / 2} x_{2}^{1}\right]+\operatorname{Re} S_{2,1}^{\prime \prime}, \\
& 0=\cos \left[(1+2 \alpha)^{1 / 2} x_{2}^{2}\right]+\operatorname{Im} S_{2,1}^{\prime \prime} .
\end{aligned}
$$

Будем искать $x_{2}^{1}, x_{2}^{2}, \lambda$ в виде

$$
\begin{gathered}
\lambda=\left(k_{0}^{2}+\tilde{\lambda}\right)^{1 / 4}, \quad x_{2}^{j}=\frac{\arccos \left(-\tilde{x}_{2}^{j}\right)}{(1+2 \alpha)^{1 / 2}}, \\
\text { где }|\tilde{\lambda}|<C \varepsilon^{1 / 4} \text { и }\left|\tilde{x}_{2}^{j}\right|<C \varepsilon^{1 / 4}, \quad j=1,2 .
\end{gathered}
$$

Тогда уравнения (4.76)-(4.78) для новых неизвестных $\tilde{\lambda}$ и $\tilde{x}_{2}^{j}$ примут вид:

$$
\begin{aligned}
\tilde{\lambda} & =\widetilde{S}_{2,2}^{\prime \prime}\left(\tilde{\lambda}, \tilde{x}_{2}^{1}, \tilde{x}_{2}^{2}\right), \\
\tilde{x}_{2}^{1} & =\operatorname{Re} \widetilde{S}_{2,1}^{\prime \prime}\left(\tilde{\lambda}, \tilde{x}_{2}^{1}, \tilde{x}_{2}^{2}\right), \\
\tilde{x}_{2}^{2} & =\operatorname{Im} \widetilde{S}_{2,1}^{\prime \prime}\left(\tilde{\lambda}, \tilde{x}_{2}^{1}, \tilde{x}_{2}^{2}\right),
\end{aligned}
$$

где

$$
\widetilde{S}_{2, j}^{\prime \prime}\left(\tilde{\lambda}, \tilde{x}_{2}^{1}, \tilde{x}_{2}^{2}\right)=S_{2, j}^{\prime \prime}\left(\left(k_{0}^{2}+\tilde{\lambda}\right)^{1 / 4}, \frac{\arccos \left(-\tilde{x}_{2}^{1}\right)}{(1+2 \alpha)^{1 / 2}}, \frac{\arccos \left(-\tilde{x}_{2}^{2}\right)}{(1+2 \alpha)^{1 / 2}}\right)
$$

при $j=1,2$. Ясно, что функции $\widetilde{S}_{2, j}^{\prime \prime}\left(\tilde{\lambda}, \tilde{x}_{2}^{1}, \tilde{x}_{2}^{2}\right)$ непрерывны при $|\tilde{\lambda}|<C \varepsilon^{1 / 4}$, $\left|\tilde{x}_{2}^{j}\right|<C \varepsilon^{1 / 4}, j=1,2$, причем модули этих функций не превосходят $C \varepsilon^{1 / 2}$. Остается применить к (4.79)-(4.81) принцип сжимающих отображений (см. [6]). Замечая, что решение (4.79)-(4.81) после перехода к переменным $x_{2}^{1}, x_{2}^{2}, \lambda$ является также решением уравнений (4.69), (4.70), убеждаемся в справедливости следуюшей теоремы.

TЕОРема 4.3. Для любого достаточно малого $\varepsilon>0$ найдутся точки $x^{1}(\varepsilon)$, $x^{2}(\varepsilon)$ и параметр $\lambda(\varepsilon)$ такие, что однородная задача (4.3), (4.4) в области

$$
\Pi(\varepsilon)=\left\{x:\left|x_{1}\right|<\pi, x_{2}>0\right\} \backslash \bigcup_{j=1}^{2}\left\{x:\left|x-x^{j}(\varepsilon)\right| \leqslant \lambda(\varepsilon)\right\}
$$

имеет нетривиальное решение в пространстве $W_{\beta}^{l+1}(\Pi(\varepsilon) ; \alpha)$ с положительным показателем $\beta$ (при этом $l$ - любое натуральное число). Иными словами, при надлежащем выборе геометрических параметров существует әкспоненциально затухающее при $x_{2} \rightarrow+\infty$ решение однородной задачи $в$ полуполосе с двумя малыми круговыми отверстиями. 


\section{§5. Обоснование}

1. Некоторые определения и вспомогательные утверждения. Введем оператор

$$
\mathfrak{L}(\mu, \delta): \mathbb{W}_{\beta, l}^{l+1}(\Pi(\mu) ; \alpha, \delta) \rightarrow \mathscr{V}_{\beta, l}^{l-1}(\Pi(\mu) ; \alpha) \times \mathcal{V}_{\beta, l}^{l-1 / 2}(\Gamma(\mu) ; \alpha)
$$

действующий по правилу

$$
\mathfrak{L}(\mu, \delta) u=\left\{L_{\delta}(\nabla) u,\left.\partial_{n} u\right|_{\Gamma(\mu)}\right\}
$$

Здесь $\delta$ и $\mu$ - малые положительные параметры, $l \in\{1,2, \ldots\}$, а $\beta>0$ - тот же весовой показатель, что и в $\S 4$. Напомним, что основная цель этого параграфа построить обратный оператор $\mathfrak{R}(\mu, \delta)$ для $\mathscr{L}(\mu, \delta)$ и оценить его норму. Для этого нам потребуются следуюшие определения и утверждения.

Пусть $\widehat{\mathbb{W}}_{\beta, l-\gamma}^{l+1}(\Pi(0) ; \alpha, 0)$ - пространство функций, представимых в виде

$$
u(x)=\sum_{j=1}^{2} u\left(x^{j}\right) \chi\left(r_{j} \delta_{1}^{-1}\right)+\tilde{u}(x)
$$

где $u\left(x^{j}\right) \in \mathbb{C}, \tilde{u} \in \widehat{\mathbb{W}}_{\beta, l-\gamma}^{l+1}(\Pi(0) ; \alpha, 0), \gamma \in(0,1)$. Естественная норма в этом пространстве такова:

$$
\left\|u ; \widehat{\mathbb{W}}_{\beta, l-\gamma}^{l+1}(\Pi(0) ; \alpha, 0)\right\|=\left(\sum_{j=1}^{2}\left|u\left(x^{j}\right)\right|^{2}+\left\|\tilde{u} ; \widehat{\mathbb{W}}_{\beta, l-\gamma}^{l+1}(\Pi(0) ; \alpha, 0)\right\|^{2}\right)^{1 / 2} .
$$

Следуюшая лемма, касающаяся разрешимости задачи

$$
\begin{aligned}
L_{0}(\nabla) u(x) & =f(x), & x \in \Pi(0), \\
\partial_{n} u(x) & =g(x), & x \in \Gamma(0),
\end{aligned}
$$

в классе $\widehat{\mathbb{W}}_{\beta, l-\gamma}^{l+1}(\Pi(0) ; \alpha, 0)$, является конкретизацией общих результатов $[7 ; \S \S 5.3,6.1]$ (см. также $[3 ; \S 1.5])$.

Лемма 5.1. При $\gamma \in(0,1) u f \in \mathcal{V}_{\beta, l-\gamma}^{l-1}(\Pi(0) ; \alpha), g \in \mathcal{V}_{\beta, l-\gamma}^{l-1 / 2}(\Gamma(0) ; \alpha) c y-$ ществует решение $u \in \widehat{\mathbb{W}}_{\beta, l-\gamma}^{l+1}(\Pi(0) ; \alpha, 0)$ задачи $(5.4),(5.5)$ и верна оценка

$$
\left\|u ; \widehat{\mathbb{W}}_{l-\gamma}^{l+1}(\Pi(0) ; \alpha, 0)\right\| \leqslant C\left(\left\|f ; \mathcal{V}_{\beta, l-\gamma}^{l-1}(\Pi(0) ; \alpha)\right\|+\left\|g ; \mathcal{V}_{\beta, l-\gamma}^{l-1}(\Gamma(0) ; \alpha)\right\|\right)
$$


ЗАмечание 5.2. Константа $C$ в неравенстве (5.6) непрерывно зависит от геометрических параметров $x^{1}$ и $x^{2}$. Далее эти параметры варьируются: величины $x_{1}^{1}, x_{2}^{1}$ остаются неизменными, а $x_{2}^{1}, x_{2}^{2}$ принадлежат некоторым (замкнутым) отрезкам. Поэтому постоянную $C$ можно выбирать не зависящей от $x^{j}$.

Напомним определение пространства Кондратьева [2] для произвольной области $\Xi \subset \mathbb{R}^{2}$. Под $V_{\sigma}^{l+1}(\Xi)$ подразумевается замыкание $C_{0}^{\infty}(\Xi \backslash\{0\})$ по норме

$$
\left\|\cdot ; V_{\sigma}^{l+1}(\Xi)\right\|=\left(\sum_{|\tau| \leqslant l+1} \int_{\Xi} r^{2(\sigma-l-1+|\tau|)}\left|\nabla^{\tau} \cdot\right|^{2} d x\right)^{1 / 2} .
$$

Естественным образом определяется пространство $V_{\sigma}^{l-1 / 2}(\partial \Xi)$ следов нормальных производных функций из $V_{\sigma}^{l+1}(\Xi)$ на $\partial \Xi$.

Рассмотрим внешнюю задачу Неймана

$$
\begin{aligned}
& -\Delta u(x)=f(x), \quad x \in \mathbb{R}^{2} \backslash D_{1}, \\
& \partial_{\nu} u(x)=g(x), \quad x \in \partial D_{1},
\end{aligned}
$$

где $D_{\rho}$ - диск с радиусом $\rho$ и центром в начале координат, $f \in V_{l+\gamma}^{l-1}\left(\mathbb{R}^{2} \backslash D_{1}\right)$, $g \in V_{l+\gamma}^{l-1 / 2}\left(\partial D_{1}\right), \gamma \in(0,1)$. Следуюшее утверждение известно (см., например, $[3 ; \S 1.5],[7 ; \S 6.4])$.

Лемма 5.3. Задача (5.7), (5.8) разрешима в пространстве $V_{\gamma}^{l+1}\left(\mathbb{R}^{2} \backslash D_{1}\right)$ в том и только том случае, если

$$
(f, 1)_{\mathbb{R}^{2} \backslash D_{1}}+(g, 1)_{\partial D_{1}}=0 .
$$

При выполнении этого условия решение $u \in V_{l+\gamma}^{l+1}\left(\mathbb{R}^{2} \backslash D_{1}\right)$ единственно и верна оченка

$$
\left\|u ; V_{l+\gamma}^{l+1}\left(\mathbb{R}^{2} \backslash D_{1}\right)\right\| \leqslant C\left(\left\|f ; V_{l+\gamma}^{l-1}\left(\mathbb{R}^{2} \backslash D_{1}\right)\right\|+\left\|g ; V_{l+\gamma}^{l-1 / 2}\left(\partial D_{1}\right)\right\|\right) .
$$

2. Оценка нормы оператора $\mathfrak{R}(\mu, 0)$. В этом разделе мы построим оператор $\mathfrak{R}(\mu, 0)$, обратный к оператору задачи

$$
\begin{aligned}
L_{0}(\nabla) u(\mu, x) & =f(\mu, x), & & x \in \Pi(\mu), \\
\partial_{n} u & =g(\mu, x), & & x \in \Gamma(\mu)
\end{aligned}
$$

(с условиями квазипериодичности и излучения), и получим оценку его нормы. Замысел заключается в следуюшем: сначала отыскивается приближенное решение задачи (5.11), (5.12) (оно склеивается из решений предельных задач (5.7), (5.8) и $(5.4),(5.5))$, а затем благодаря малости его невязки в (5.11), (5.12) обратный оператор получается как ряд Неймана. Из этого представления вытекает и оценка нормы $\mathfrak{R}(\mu, 0)$. 
Запишем правые части $(5.10),(5.11)$ в виде

$$
\begin{aligned}
& f(\mu, x)=\mu^{-2}\left(f_{1}\left(\mu, \xi^{1}\right)+f_{2}\left(\mu, \xi^{2}\right)\right)+f_{3}(\mu, x) \\
& g(\mu, x)=\mu^{-1}\left(g_{1}\left(\mu, \xi^{1}\right)+g_{2}\left(\mu, \xi^{2}\right)\right)+g_{3}(\mu, x)
\end{aligned}
$$

где $\xi^{j}=\mu^{-1}\left(x-x^{j}\right)$

$$
\begin{gathered}
f_{3}(\mu, x)=\left(1-\chi\left(r_{1} \mu^{-1 / 2}\right)\right)\left(1-\chi\left(r_{2} \mu^{-1 / 2}\right)\right) f(\mu, x) \\
g_{3}(\mu, x)=\left.g(\mu, x)\right|_{\Gamma(0)}, \\
f_{j}\left(\mu, \xi^{j}\right)=\chi\left(r_{j} \mu^{-1 / 2}\right) \mu^{2} f(\mu, x) \\
g_{j}\left(\mu, \xi^{j}\right)=\left.\mu g\left(\mu, \xi^{j}\right)\right|_{\partial D_{1}}, \quad j=1,2 .
\end{gathered}
$$

Подчеркнем, что срезки, фигурирующие в (5.15) и (5.16), образуют разбиение единицы. Непосредственно проверяются оценки

$$
\begin{aligned}
& \left\|f_{3} ; \mathscr{V}_{\beta, l}^{l-1}(\Pi(0) ; \alpha)\right\|+\sum_{j=1}^{2}\left\|f_{j} ; V_{l}^{l-1}\left(\mathbb{R}^{2} \backslash D_{1}\right)\right\| \leqslant C\left\|f ; \mathscr{V}_{\beta, l}^{l-1}(\Pi(\mu) ; \alpha)\right\|, \\
& \left\|g_{3} ; \mathscr{V}_{\beta, l}^{l-1}(\Gamma(0) ; \alpha)\right\|+\sum_{j=1}^{2}\left\|g_{j} ; V_{l}^{l-1 / 2}\left(\partial D_{1}\right)\right\| \leqslant C\left\|g ; \mathscr{V}_{\beta, l}^{l-1 / 2}(\Gamma(\mu) ; \alpha)\right\| .
\end{aligned}
$$

Представления (5.13), (5.14) приспособлены к возникаюшему набору предельных задач. Так, $f_{3}$ и $g_{3}$ будут компенсированы при решении основной предельной задачи (5.4), (5.5). Функции $f_{1}, g_{1}$ и $f_{2}, g_{2}$ направляются в правые части предельных задач, описьвающих явление пограничного слоя:

$$
\begin{array}{rlrl}
-\Delta_{\xi} u_{j}\left(\mu, \xi^{j}\right) & =f_{j}\left(\mu, \xi^{j}\right), & & \xi^{j} \in \mathbb{R}^{2} \backslash D_{1}, \\
\partial_{\nu} u_{j}\left(\mu, \xi^{j}\right) & =g_{j}\left(\mu, \xi^{j}\right)+A_{j}, & \xi^{j} \in \partial D_{1} .
\end{array}
$$

Здесь $\partial_{\nu}-$ производная вдоль внешней нормали, индекс $j$ принимает значения 1,2 , а дополнительное слагаемое $A_{j}$ используется для соблюдения условий разрешимости задач в классе $V_{l+\gamma}^{l+1}\left(\mathbb{R}^{2} \backslash D_{1}\right), \gamma \in(0,1)$,

$$
0=\left(f_{j}, 1\right)_{\mathbb{R}^{2} \backslash D_{1}}+\left(g_{j}+A_{j}, 1\right)_{\partial D_{1}}
$$

(см. лемму 5.3), т.е.

$$
A_{j}=-(2 \pi)^{-1}\left(\left(f_{j}, 1\right)_{\mathbb{R}^{2} \backslash D_{1}}+\left(g_{j}, 1\right)_{\partial D_{1}}\right) .
$$

Для решения $u_{j}$ задачи $(5.17),(5.18)$ верна вытекаюшая из (5.10) оценка

$$
\begin{aligned}
& \left\|u_{j}\left(\mu, \xi^{j}\right) ; V_{l+\gamma}^{l+1}\left(\mathbb{R}^{2} \backslash D_{1}\right)\right\| \\
& \quad \leqslant C\left(\left\|f_{j}\left(\mu, \xi^{j}\right) ; V_{l+\gamma}^{l-1}\left(\mathbb{R}^{2} \backslash D_{1}\right)\right\|+\left\|g_{j}\left(\mu, \xi^{j}\right) ; V_{l+\gamma}^{l-1 / 2}\left(\partial D_{1}\right)\right\|\right) .
\end{aligned}
$$


Обращаем внимание на то, что правая часть уравнения (5.19) финитна и потому попадает в пространство $V_{l+\gamma}^{l-1}\left(\mathbb{R}^{2} \backslash D_{1}\right)$ с произвольньм показателем $\gamma$. Кроме того, поскольку

$$
f_{j}\left(\mu, \xi^{j}\right)=0 \text { при }\left|\xi^{j}\right|>c \mu^{-1 / 2}
$$

в случае $\gamma \in[0,1]$ верна оценка

$$
\left\|f_{j}\left(\mu, \xi^{j}\right) ; V_{l+\gamma}^{l-1}\left(\mathbb{R}^{2} \backslash D_{1}\right)\right\| \leqslant c \mu^{-\gamma / 2}\left\|f_{j}\left(\mu, \xi^{j}\right) ; V_{l}^{l-1}\left(\mathbb{R}^{2} \backslash D_{1}\right)\right\| .
$$

Положим

$$
R_{j}\{f, g\}=u_{j}\left(\mu, \xi^{j}\right) \chi\left(r_{j} \mu^{-1 / 4}\right) .
$$

Равенство (5.25), записанное в переменных $\left(x_{1}, x_{2}\right)$, определяет линейный непрерывный оператор из $\mathscr{V}_{\beta, l}^{l-1}(\Pi(\mu) ; \alpha) \times \mathscr{V}_{\beta, l}^{l-1 / 2}(\Gamma(\mu) ; \alpha)$ в $\mathbb{W}_{\beta, l}^{l+1}(\Pi(\mu) ; \alpha, 0)$. Действительно, из определения нормы в пространстве $\mathbb{W}_{\beta, l}^{l+1}(\Pi(\mu) ; \alpha, 0)$ и равенства (5.25) следует, что

$$
\left\|R_{j}\{f, g\} ; \mathbb{W}_{\beta, l}^{l+1}(\Pi(\mu) ; \alpha, 0)\right\| \leqslant C\left\|u_{j}\left(\mu, \xi^{j}\right) ; V_{l}^{l+1}\left(\mathbb{R}^{2} \backslash D_{1}\right)\right\| .
$$

Пользуясь очевидньм неравенством

$$
\left\|u_{j}\left(\mu, \xi^{j}\right) ; V_{l}^{l+1}\left(\mathbb{R}^{2} \backslash D_{1}\right)\right\| \leqslant C\left\|u_{j}\left(\mu, \xi^{j}\right) ; V_{l+\gamma}^{l+1}\left(\mathbb{R}^{2} \backslash D_{1}\right)\right\|
$$

и оценками $(5.23),(5.24)$, заключаем, что правая часть (5.26) менњше

$$
C \mu^{-\gamma / 2}\left(\left\|f_{j}\left(\mu, \xi^{j}\right) ; V_{l}^{l-1}\left(\mathbb{R}^{2} \backslash D_{1}\right)\right\|+\left\|g_{j}\left(\mu, \xi^{j}\right) ; V_{l}^{l-1 / 2}\left(\partial D_{1}\right)\right\|\right),
$$

причем мажоранту этого выражения дают неравенства (5.17), (5.18). Из вышесказанного вытекает оценка

$$
\begin{aligned}
& \left\|R_{j}\{f, g\} ; \mathbb{W}_{\beta, l}^{l+1}(\Pi(\mu) ; \alpha, 0)\right\| \\
& \quad \leqslant C \mu^{-\gamma / 2}\left(\left\|f ; \mathscr{V}_{\beta, l}^{l-1}(\Pi(\mu) ; \alpha)\right\|+\left\|g ; \mathscr{V}_{\beta, l}^{l-1 / 2}(\Gamma(\mu) ; \alpha)\right\|\right),
\end{aligned}
$$

гарантируюшая ограниченность оператора $R_{j}$. Подставляя $R_{j}\{f, g\}$ в (5.11), получаем

$$
\begin{aligned}
L_{0}(\nabla) R_{j}\{f, g\} & =-\mu^{-2} \Delta_{\xi}\left(u_{j}\left(\mu, \xi^{j}\right) \chi\left(\mu^{3 / 4}\left|\xi^{j}\right|\right)\right)-k_{0}^{2} u_{j}\left(\mu, \xi^{j}\right) \chi\left(\mu^{3 / 4}\left|\xi^{j}\right|\right) \\
& =f \chi\left(\mu^{-1 / 2}\right)+\mathscr{S}_{j}^{(1)}\{f, g\}
\end{aligned}
$$

где

$$
\mathscr{S}_{j}^{(1)}\{f, g\}=-\mu^{-2}\left[\Delta_{\xi}, \chi\left(\mu^{3 / 4}\left|\xi^{j}\right|\right)\right] u_{j}\left(\mu, \xi^{j}\right)-k_{0}^{2} u_{j}\left(\mu, \xi^{j}\right) \chi\left(\mu^{3 / 4}\left|\xi^{j}\right|\right) .
$$

Очевидно, что равенство (5.29), записанное в переменных $\left(x_{1}, x_{2}\right)$, определяет линейный непрерьвньй оператор из $\mathcal{V}_{\beta, l}^{l-1}(\Pi(\mu) ; \alpha) \times \mathcal{V}_{\beta, l}^{l-1 / 2}(\Gamma(\mu) ; \alpha)$ в 
$\mathscr{V}_{\beta, l}^{l-1}(\Pi(\mu) ; \alpha)$, который мы будем обозначать через $\mathscr{S}_{j}^{(1)}$ (напомним, что функция $u_{j}$ строилась по $\{f, g\}$ - элементу пространства $\left.\mathcal{V}_{\beta, l}^{l-1}(\Pi(\mu) ; \alpha) \times \mathscr{V}_{\beta, l}^{l-1 / 2}(\Gamma(\mu) ; \alpha)\right)$. Покажем, что норма $\mathscr{S}_{j}^{(1)}$ мала. Рассмотрим норму второго слагаемого в (5.29) справа. Сначала, используя определения норм весовых классов и расположение носителя срезки, убеждаемся в том, что

$$
\begin{aligned}
& \left\|k_{0}^{2} u_{j}\left(\mu, \xi^{j}\right) \chi\left(\mu^{3 / 4}\left|\xi^{j}\right|\right) ; \mathfrak{V}_{\beta, l}^{l-1}(\Pi(\mu) ; \alpha)\right\| \\
& \quad \leqslant C \mu^{2}\left\|k_{0}^{2} u_{j}\left(\mu, \xi^{j}\right) \chi\left(\mu^{3 / 4}\left|\xi^{j}\right|\right) ; V_{l}^{l-1}\left(D_{2 \mu^{-3 / 4}} \backslash D_{1}\right)\right\| \\
& \quad \leqslant C \mu^{2}\left\|k_{0}^{2} u_{j}\left(\mu, \xi^{j}\right) \chi\left(\mu^{3 / 4}\left|\xi^{j}\right|\right) ; V_{l+2}^{l+1}\left(D_{2 \mu^{-3 / 4}} \backslash D_{1}\right)\right\| \\
& \quad \leqslant C \mu^{(2+3 \gamma) / 4}\left\|u_{j}\left(\mu, \xi^{j}\right) ; V_{l+\gamma}^{l+1}\left(\mathbb{R}^{2} \backslash D_{1}\right)\right\| .
\end{aligned}
$$

Затем, оценивая правую часть (5.30) в соответствии с неравенствами (5.23), (5.24), заключаем, что

$$
\begin{aligned}
& C \mu^{(2+3 \gamma) / 4}\left\|u_{j}\left(\mu, \xi^{j}\right) ; V_{l+\gamma}^{l+1}\left(\mathbb{R}^{2} \backslash D_{1}\right)\right\| \\
& \quad \leqslant C \mu^{(2+\gamma) / 4}\left(\left\|f_{j}\left(\mu, \xi^{j}\right) ; V_{l}^{l-1}\left(\mathbb{R}^{2} \backslash D_{1}\right)\right\|+\left\|g_{j}\left(\mu, \xi^{j}\right) ; V_{l}^{l-1 / 2}\left(\partial D_{1}\right)\right\|\right) .
\end{aligned}
$$

И наконец, выражения (5.30), (5.31) позволяют найти требуемую оценку

$$
\begin{aligned}
& \left\|k_{0}^{2} u_{j}\left(\mu, \xi^{j}\right) \chi\left(\mu^{3 / 4}\left|\xi^{j}\right|\right) ; \mathscr{V}_{\beta, l}^{l-1}(\Pi(\mu) ; \alpha)\right\| \\
& \quad \leqslant C \mu^{(2+\gamma) / 4}\left(\left\|f_{j}\left(\mu, \xi^{j}\right) ; V_{l}^{l-1}\left(\mathbb{R}^{2} \backslash D_{1}\right)\right\|+\left\|g_{j}\left(\mu, \xi^{j}\right) ; V_{l}^{l-1 / 2}\left(\partial D_{1}\right)\right\|\right) .
\end{aligned}
$$

Перейдем к рассмотрению нормы первого слагаемого в (5.29). Как и выше, пользуясь определением норм весовых классов, выводим

$$
\begin{aligned}
& \left\|\mu^{-2}\left[\Delta, \chi\left(\mu^{3 / 4}\left|\xi^{j}\right|\right)\right] u_{j}\left(\mu, \xi^{j}\right) ; \mathcal{V}_{\beta, l}^{l-1}(\Pi(\mu) ; \alpha)\right\| \\
& \quad \leqslant C\left\|\left[\Delta, \chi\left(\mu^{3 / 4}\left|\xi^{j}\right|\right)\right] u_{j}\left(\mu, \xi^{j}\right) ; V_{l}^{l-1}\left(D_{2 \mu^{-3 / 4}} \backslash D_{1}\right)\right\| .
\end{aligned}
$$

Так как носители коэффициентов коммутатора $\left[\Delta, \chi\left(\mu^{3 / 4}\left|\xi^{j}\right|\right)\right]$ расположены в кольце $\bar{D}_{2 \mu^{-3 / 4}} \backslash D_{\mu^{-3 / 4}}$, правая часть (5.33) может быть оценена следующим образом:

$$
\begin{aligned}
& \left\|\left[\Delta, \chi\left(\mu^{3 / 4}\left|\xi^{j}\right|\right)\right] u_{j}\left(\mu, \xi^{j}\right) ; V_{l}^{l-1}\left(D_{2 \mu^{-3 / 4}} \backslash D_{1}\right)\right\| \\
& \quad \leqslant C\left\|u_{j}\left(\mu, \xi^{j}\right) ; V_{l}^{l+1}\left(D_{2 \mu^{-3 / 4}} \backslash D_{\mu^{-3 / 4}}\right)\right\| \\
& \quad \leqslant C \mu^{3 \gamma / 4}\left\|u_{j}\left(\mu, \xi^{j}\right) ; V_{l+\gamma}^{l+1}\left(\mathbb{R}^{2} \backslash D_{1}\right)\right\| .
\end{aligned}
$$

Обработаем правую часть (5.34). Последовательно применяя неравенства (5.23) и (5.24), имеем

$$
\begin{aligned}
& C \mu^{3 \gamma / 4}\left\|u_{j}\left(\mu, \xi^{j}\right) ; V_{l+\gamma}^{l+1}\left(\mathbb{R}^{2} \backslash D_{1}\right)\right\| \\
& \quad \leqslant C \mu^{3 \gamma / 4}\left(\left\|f_{j}\left(\mu, \xi^{j}\right) ; V_{l+\gamma}^{l-1}\left(\mathbb{R}^{2} \backslash D_{1}\right)\right\|+\left\|g_{j}\left(\mu, \xi^{j}\right) ; V_{l+\gamma}^{l-1 / 2}\left(\partial D_{1}\right)\right\|\right) \\
& \quad \leqslant C \mu^{\gamma / 4}\left(\left\|f_{j}\left(\mu, \xi^{j}\right) ; V_{l}^{l-1}\left(\mathbb{R}^{2} \backslash D_{1}\right)\right\|+\left\|g_{j}\left(\mu, \xi^{j}\right) ; V_{l}^{l-1 / 2}\left(\partial D_{1}\right)\right\|\right) .
\end{aligned}
$$


Финальная оценка первого слагаемого в (5.29)

$$
\begin{aligned}
& \left\|\mu^{-2}\left[\Delta, \chi\left(\mu^{3 / 4}\left|\xi^{j}\right|\right)\right] u_{j}\left(\mu, \xi^{j}\right) ; \mathscr{V}_{\beta, l}^{l-1}(\Pi(\mu) ; \alpha)\right\| \\
& \quad \leqslant C \mu^{\gamma / 2}\left(\left\|f_{j}\left(\mu, \xi^{j}\right) ; V_{l}^{l-1}\left(\mathbb{R}^{2} \backslash D_{1}\right)\right\|+\left\|g_{j}\left(\mu, \xi^{j}\right) ; V_{l}^{l-1 / 2}\left(\partial D_{1}\right)\right\|\right)
\end{aligned}
$$

следует из неравенств (5.33), (5.34) и (5.35).

Вспоминая (5.29) и складьвая неравенства (5.32) и (5.36), получаем

$$
\begin{aligned}
& \left\|\mathscr{S}_{j}^{(1)}\{f, g\} ; \mathscr{V}_{\beta, l}^{l-1}(\Pi(\mu) ; \alpha)\right\| \\
& \quad \leqslant C \mu^{\gamma / 2}\left(\left\|f_{j}\left(\mu, \xi^{j}\right) ; V_{l}^{l-1}\left(\mathbb{R}^{2} \backslash D_{1}\right)\right\|+\left\|g_{j}\left(\mu, \xi^{j}\right) ; V_{l}^{l-1 / 2}\left(\partial D_{1}\right)\right\|\right) .
\end{aligned}
$$

Осталось оценить правую часть (5.37) согласно неравенствам (5.17), (5.18), именно,

$$
\begin{aligned}
& \left\|\mathscr{S}_{j}^{(1)}\{f, g\} ; \mathscr{V}_{\beta, l}^{l-1}(\Pi(\mu) ; \alpha)\right\| \\
& \quad \leqslant C \mu^{\gamma / 2}\left(\left\|f ; \mathscr{V}_{\beta, l}^{l-1}(\Pi(\mu) ; \alpha)\right\|+\left\|g ; \mathscr{V}_{\beta, l}^{l-1 / 2}(\Gamma(\mu) ; \alpha)\right\|\right) .
\end{aligned}
$$

Перейдем к рассмотрению внешней предельной задачи в полосе П $(0)$. Из леммы 5.1 вытекает сушествование решения $u_{3} \in \widehat{\mathbb{W}}_{\beta, l-\gamma}^{l+1}(\Pi(0) ; \alpha, 0)$ следуюшей задачи:

$$
\begin{aligned}
L_{0}(\nabla) u_{3} & =f_{3}, \quad x \in \Pi(0), \\
\partial_{n} u_{3} & =g_{3}, \quad x \in \Gamma(0) ;
\end{aligned}
$$

при этом

$$
\begin{aligned}
& \left\|u_{3} ; \widehat{\mathbb{W}}_{\beta, l-\gamma}^{l+1}(\Pi(0) ; \alpha, 0)\right\| \\
& \quad \leqslant C\left(\left\|f_{3} ; \mathscr{V}_{\beta, l-\gamma}^{l-1}(\Pi(0) ; \alpha)\right\|+\left\|g_{3} ; \mathcal{V}_{\beta, l-\gamma}^{l-1 / 2}(\Gamma(0) ; \alpha)\right\|\right) .
\end{aligned}
$$

Отметим, что носители правых частей уравнений (5.39), (5.40) отделены от точек $x^{1}, x^{2}$, и потому пара $\left(f_{3}, g_{3}\right)$ попадает в пространство

$$
\mathscr{V}_{\beta, l-\gamma}^{l-1}(\Pi(0) ; \alpha) \times \mathscr{V}_{\beta, l-\gamma}^{l-1 / 2}(\Gamma(0) ; \alpha)
$$

с произвольным показателем $\gamma$. Более того, поскольку

$$
f_{3}(\mu, x)=0 \text { при }\left|r_{j}\right|<c \mu^{-1 / 2}, \quad j=1,2,
$$

при $\gamma \in[0,1]$ верна оценка

$$
\begin{aligned}
& \left\|f_{3} ; \mathscr{V}_{\beta, l-\gamma}^{l-1}(\Pi(0) ; \alpha)\right\|+\left\|g_{3} ; \mathcal{V}_{\beta, l-\gamma}^{l-1 / 2}(\Gamma(0) ; \alpha)\right\| \\
& \quad \leqslant C \mu^{-\gamma / 2}\left(\left\|f_{3} ; \mathscr{V}_{\beta, l-\gamma}^{l-1}(\Pi(0) ; \alpha)\right\|+\left\|g_{3} ; \mathcal{V}_{\beta, l-\gamma}^{l-1}(\Gamma(0) ; \alpha)\right\|\right) .
\end{aligned}
$$


Найдем мажоранту нормы $u_{3}$ в пространстве $\mathbb{W}_{\beta, l}^{l+1}(\Pi(\mu) ; \alpha, 0)$. Обращаем внимание на то, что из-за своего поведения в точках $x^{j}$ функция $u_{3}$ может не принадлежать пространству $\mathbb{W}_{\beta, l}^{l+1}(\Pi(\mu) ; \alpha, 0)$ в предельной области П(0). Действительно, в силу (5.2) включение $u_{3} \in \widehat{\mathbb{W}}_{\beta, l-\gamma}^{l+1}(\Pi(0) ; \alpha, 0)$ означает, что

$$
u_{3}(x)=\sum_{j=1}^{2} u_{3}\left(x^{j}\right) \chi\left(r_{j} \delta_{1}^{-1}\right)+\tilde{u}_{3}(x), \quad \tilde{u}_{3}(x) \in \mathbb{W}_{\beta, l-\gamma}^{l+1}(\Pi(0) ; \alpha, 0)
$$

т.е. $u_{3} \in \mathbb{W}_{\beta, l}^{l+1}(\Pi(0) ; \alpha, 0)$ лиш при условии $u\left(x^{j}\right)=0$. Тем не менее, из представления (5.43), определения норм весовых классов и формулы (5.3) вытекает следующая цепочка неравенств:

$$
\begin{aligned}
& \left\|u_{3} ; \mathbb{W}_{\beta, l}^{l+1}(\Pi(\mu) ; \alpha, 0)\right\| \\
& \quad \leqslant C\left(\sum_{j=1}^{2}\left|u_{3}\left(x^{j}\right)\right|\left\|1 ; \mathbb{W}_{\beta, l}^{l+1}\left(D_{\delta_{1}}^{j} \backslash D_{\mu}^{j} ; \alpha, 0\right)\right\|+\left\|\tilde{u}_{3} ; \mathbb{W}_{\beta, l}^{l+1}(\Pi(\mu) ; \alpha, 0)\right\|\right) \\
& \quad \leqslant C\left(\sum_{j=1}^{2}\left|u_{3}\left(x^{j}\right)\right||\ln \mu|^{1 / 2}+\left\|\tilde{u}_{3} ; \mathbb{W}_{\beta, l-\gamma}^{l+1}(\Pi(\mu) ; \alpha, 0)\right\|\right) .
\end{aligned}
$$

Здесь мы использовали тот факт, что

$$
\left\|1 ; \mathbb{W}_{\beta, l}^{l+1}\left(D_{\delta_{1}}^{j} \backslash D_{\mu}^{j} ; \alpha, 0\right)\right\| \sim\left(\int_{\mu}^{\delta_{1}} r_{j}^{2[l-(l+1)]} r_{j} d r_{j}\right)^{1 / 2} \sim|\ln \mu|^{1 / 2}
$$

Осталось заметить, что неравенства $(5.41),(5.42),(5.44)$ и $(5.17),(5.18)$ влекут искомую оценку

$$
\left\|u_{3} ; \mathbb{W}_{\beta, l}^{l+1}(\Pi(\mu) ; \alpha, 0)\right\| \leqslant C \mu^{-\gamma}\left(\left\|f ; \mathscr{V}_{\beta, l}^{l-1}(\Pi(0) ; \alpha)\right\|+\left\|g ; \mathscr{V}_{\beta, l}^{l-1}(\Gamma(0) ; \alpha)\right\|\right)
$$

Введем линейный оператор $R_{3}$, действующий из $\mathcal{V}_{\beta, l}^{l-1}(\Pi(\mu) ; \alpha) \times \mathcal{V}_{\beta, l}^{l-1 / 2}(\Gamma(\mu) ; \alpha)$ в $\mathbb{W}_{\beta, l}^{l+1}(\Pi(\mu) ; \alpha, 0)$ по правилу:

$$
R_{3}\{f, g\}=u_{3}
$$

Происхождение функции $u_{3}$ и соотношения $(5.15)$, (5.16) убеждают нас в корректности этого определения. Непрерьвность оператора $R_{3}$ следует из неравенства (5.45).

Подставим в $(5.11),(5.12)$ функцию $\sum_{p=1}^{3} R_{p}\{f, g\}$. Ясно, что она оставляет малую невязку в уравнении (5.11) (см. (5.28), (5.39) и оценку (5.38)), в то же время невязка в (5.12) может оказаться большой. Действительно, вычисляя значение нормальной производной от $\sum_{p=1}^{3} R_{p}\{f, g\}$ при $x \in \partial D_{\mu}^{j}$, получаем

$$
\partial_{n} \sum_{p=1}^{3} R_{p}\{f, g\}=\mu^{-1} \partial_{\nu} u_{j}+\partial_{n} u_{3}=g+\mu^{-1} A_{j}+\partial_{n} \tilde{u}_{3} \quad \text { на } D_{\mu}^{j} \text {. }
$$


Покажем, что норма третьего слагаемого в (5.47) справа мала. В самом деле,

$$
\begin{aligned}
& \left\|\partial_{n} \tilde{u}_{3} ; \mathscr{V}_{\beta, l}^{l-1 / 2}\left(\partial D_{\mu}^{j}\right)\right\| \\
& \quad \leqslant \mu^{\gamma}\left\|\partial_{n} \tilde{u}_{3} ; \mathcal{V}_{\beta, l-\gamma}^{l-1 / 2}\left(\partial D_{\mu}^{j}\right)\right\| \leqslant \mu^{\gamma}\left\|\tilde{u}_{3} ; \mathbb{W}_{\beta, l-\gamma}^{l+1}(\Pi(\mu) ; \alpha, 0)\right\| \\
& \quad \leqslant C \mu^{\gamma}\left(\left\|f_{3} ; \mathscr{V}_{\beta, l-\gamma}^{l-1}(\Pi(0) ; \alpha)\right\|+\left\|g_{3} ; \mathscr{V}_{\beta, l-\gamma}^{l-1}(\Gamma(0) ; \alpha)\right\|\right) \\
& \quad \leqslant C \mu^{\gamma / 2}\left(\left\|f_{3} ; \mathscr{V}_{\beta, l}^{l-1}(\Pi(0) ; \alpha)\right\|+\left\|g_{3} ; \mathscr{V}_{\beta, l}^{l-1}(\Gamma(0) ; \alpha)\right\|\right) .
\end{aligned}
$$

Таким образом, большая величина невязки в граничном условии обязана своим происхождением второму слагаемому в (5.47) справа. Для его компенсации необходимо добавить к $\sum_{p=1}^{3} R_{p}\{f, g\}$ некий поправочный член. В п. $3 \S 4$ были введены аналоги функции Грина $G_{j 0}, j=1,2$. В силу (4.20) они представимы в виде

$$
G_{j 0}=-\frac{1}{2 \pi} \sum_{p=1}^{2}\left(\delta_{j, p} \ln r_{p}+G_{j, 0}^{0, p}\right) \chi\left(r_{p} \delta_{1}^{-1}\right)+\widetilde{G}_{j, 0}
$$

где $\widetilde{G}_{j, 0} \in \mathbb{W}_{\beta, l-\gamma}^{l+1}(\Pi(0) ; \alpha, 0), \gamma \in(0,1)$. Рассуждения, аналогичные использованным при вьводе неравенства (5.44) и примененные к функциям $G_{j 0}, j=1,2$, показывают, что

$$
\left\|G_{j 0} ; \mathbb{W}_{\beta, l}^{l+1}(\Pi(\mu) ; \alpha, 0)\right\| \leqslant \mu^{-\gamma / 2} C .
$$

Вычислим нормальную производную от $G_{j 0}$ при $x \in \partial D_{\mu}^{m}$. Имеем

$$
\partial_{n} G_{j 0}=(2 \pi)^{-1} \mu^{-1} \delta_{j, m}+\partial_{m} \widetilde{G}_{j 0} \text { на } \partial D_{\mu}^{m} .
$$

Рассуждая так же, как при выводе (5.48), заключаем, что

$$
\left\|\partial_{n} \widetilde{G}_{j 0} ; \mathscr{V}_{\beta, l}^{l-1 / 2}\left(\partial D_{\mu}^{m}\right)\right\| \leqslant \mu^{\gamma}\left\|\partial_{n} \widetilde{G}_{j 0} ; \mathscr{V}_{\beta-\gamma, l}^{l-1 / 2}\left(\partial D_{\mu}^{m}\right)\right\| \leqslant \mu^{\gamma} C
$$

Поэтому естественно определить оператор, представляющий приближенное решение задачи (5.11), (5.12), следуюшим образом:

$$
\widetilde{R}=\sum_{j=1}^{3} R_{j}-2 \pi \sum_{j=1}^{2} A_{j} G_{j 0}
$$

ЗАмечАниЕ 5.4. Из (5.22) следует, что $A_{j}$ суть линейные функционалы от $\{f, g\}$

$$
\left|A_{j}\right| \leqslant C \mu^{-\gamma / 2}\left(\left\|f ; \mathscr{V}_{\beta, l}^{l-1}(\Pi(0) ; \alpha)\right\|+\left\|g ; \mathscr{V}_{\beta, l}^{l-1}(\Gamma(0) ; \alpha)\right\|\right)
$$

Собирая приведенные оценки (5.27), (5.45), (5.50) и (5.54), а также учитывая (5.53), приходим к неравенству для нормы оператора $\widetilde{R}$

$$
\begin{aligned}
& \left\|\widetilde{R}\{f, g\} ; \mathbb{W}_{\beta, l}^{l+1}(\Pi(\mu) ; \alpha, 0)\right\| \\
& \quad \leqslant C \mu^{-\gamma}\left(\left\|f ; \mathscr{V}_{\beta, l}^{l-1}(\Pi(\mu) ; \alpha)\right\|+\left\|g ; \mathscr{V}_{\beta, l}^{l-1 / 2}(\Gamma(\mu) ; \alpha)\right\|\right) .
\end{aligned}
$$


Принимая во внимание формулы $(5.28),(5.39)$ и то, что функции $G_{j 0}$ удовлетворяют однородному уравнению (5.11) при $x \in \Pi(\mu), \mu>0$ (см. п. $3 \S 4)$, выводим равенство

$$
L_{0}(\nabla) \widetilde{R}\{f, g\}=f+\mathscr{S}^{(1)}(\mu)\{f, g\} \text { на } \Pi(\mu),
$$

где

$$
\mathscr{S}^{(1)}(\mu)\{f, g\}=\sum_{j=1}^{2} \mathscr{S}_{j}^{(1)}(\mu)\{f, g\},
$$

и, следовательно,

$$
\left\|\mathscr{S}^{(1)}: \mathscr{V}_{\beta, l}^{l-1}(\Pi(\mu) ; \alpha) \times \mathscr{V}_{\beta, l}^{l-1 / 2}(\Gamma(\mu) ; \alpha) \rightarrow \mathscr{V}_{\beta, l}^{l-1 / 2}(\Gamma(\mu) ; \alpha)\right\| \leqslant C \mu^{\gamma / 2} .
$$

Кроме того, производные вдоль внешней нормали от функции $G_{j 0}$ обрашаются в нуль при $x \in \Gamma(0)$, а значит, в силу (5.40)

$$
\partial_{n} \widetilde{R}\{f, g\}=g \text { на } \Gamma(0) .
$$

Из формул (5.47), (5.51) и определения (5.53) вытекает, что

$$
\partial_{n} \widetilde{R}\{f, g\}=g+\mathscr{S}_{j}^{(2)}\{f, g\} \text { на } \partial D_{\mu}^{j}, \quad j=1,2 .
$$

Здесь

$$
\mathscr{S}_{j}^{(2)}\{f, g\}=\partial_{n} \tilde{u}_{3}-2 \pi A_{j} \partial_{n} \widetilde{G}_{j 0} \quad \text { на } \partial D_{\mu}^{j}, \quad j=1,2 .
$$

Оценки (5.48), (5.52) и (5.54) дают следуюшую оценку нормы оператора $\mathscr{S}_{j}^{(2)}$ :

$$
\left\|\mathscr{S}_{j}^{(2)}: \mathscr{V}_{\beta, l}^{l-1}(\Pi(\mu) ; \alpha) \times \mathscr{V}_{\beta, l}^{l-1 / 2}(\Gamma(\mu) ; \alpha) \rightarrow \mathscr{V}_{\beta, l}^{l-1 / 2}\left(\partial D_{\mu}^{j}\right)\right\| \leqslant C \mu^{\gamma / 2} .
$$

Следовательно, верно представление

$$
\partial_{n} \widetilde{R}\{f, g\}=g+\mathscr{S}^{(2)}\{f, g\} \text { на } \Gamma(\mu),
$$

где

$$
\left\|\mathscr{S}^{(2)}: \mathscr{V}_{\beta, l}^{l-1}(\Pi(\mu) ; \alpha) \times \mathscr{V}_{\beta, l}^{l-1 / 2}(\Gamma(\mu) ; \alpha) \rightarrow \mathscr{V}_{\beta, l}^{l-1 / 2}(\Gamma(\mu) ; \alpha)\right\| \leqslant C \mu^{\gamma / 2} .
$$

Из (5.63) и (5.57) вытекает равенство

$$
\mathfrak{L}(\mu, 0) \widetilde{R}=\mathbf{1}+\left\{\mathscr{S}^{(1)}, \mathscr{S}^{(2)}\right\},
$$

где $\mathbf{1}$ - тождественный оператор на $\mathscr{V}_{\beta, l}^{l-1}(\Pi(\mu) ; \alpha) \times \mathscr{V}_{\beta, l}^{l-1 / 2}(\Gamma(\mu) ; \alpha)$. Осталось положить

$$
\mathfrak{R}(\mu, 0)=\widetilde{R}\left(\mathbf{1}+\left\{\mathscr{S}^{(1)}, \mathscr{S}^{(2)}\right\}\right)^{-1}
$$

(обратный оператор понимается как сумма ряда Неймана, сходяшегося в силу оценок (5.58) и (5.64)). Итак, доказана следуюшая 
ЛЕмма 5.5. Существует такая постоянная $\mu_{1}>0$, что при любом положсительном $\mu<\mu_{1}$ оператор $\mathfrak{L}(\mu, 0)$ обратим, причем для нормы обратного оператора справедлива оценка

$$
\left\|\Re(\mu, 0): \mathscr{V}_{\beta, l}^{l-1}(\Pi(\mu) ; \alpha) \times \mathcal{V}_{\beta, l}^{l-1 / 2}(\Gamma(\mu) ; \alpha) \rightarrow \mathbb{W}_{\beta, l}^{l+1}(\Pi(\mu) ; \alpha, 0)\right\| \leqslant C_{\gamma} \mu^{-\gamma}
$$

с константой $C_{\gamma}$, не зависящей от $\mu$ и $x^{1}, x^{2}$.

3. "Непрерывные" условия излучения. В этом разделе мы докажем существование и найдем оценку нормы оператора $\mathfrak{R}(\mu, \delta)$ при малых положительных $\delta$. Попытка при помощи стандартных методов теории возмущений распространить результаты предыдущего раздела (в нем $\delta=0$ ) на положительные значения $\delta$ наталкивается на следующее препятствие: использованные нами естественные условия излучения не являются непрерывными в точке $\delta=0$, т.е. конечномерное подпространство в $\mathbb{W}_{\beta, l}^{l+1}(\Pi(\mu) ; \alpha, \delta)$, содержашее волны, которые не затухают на бесконечности, изменяется скачкообразно. Поэтому план ближайших действий содержит два этапа: сначала вводятся искусственные "непрерывные" условия излучения, приводящие к плавньм изменениям характеристик при $\delta \rightarrow+0$, и строится обратный $\mathbf{R}(\mu, \delta)$ к оператору задачи на соответствующем пространстве $\mathbf{W}_{\beta, l}^{l+1}(\Pi(\mu) ; \alpha, \delta)$, а затем $\mathbf{R}(\mu, \delta)$ переделывается в $\mathfrak{R}(\mu, \delta)$ посредством алгебраических операций на конечномерных линеалах.

"Уходящие" волны, порождающие искусственные условия излучения, зададим так:

$$
\begin{gathered}
\mathbf{u}_{2, \delta}^{-}(x)=\frac{1+i}{\sqrt{8}}\left(\left(\delta-\delta^{-1}\right) u_{2}^{+}(x)-i\left(\delta+\delta^{-1}\right) u_{2}^{-}(x)\right)\left(1-\chi\left(x_{2} R^{-1}\right)\right) \\
\mathbf{u}_{1, \delta}^{-}(x)=u_{1}^{-}(x)\left(1-\chi\left(x_{2} R^{-1}\right)\right)
\end{gathered}
$$

Обе волны определены на П $(\mu)$, причем $u_{j}^{ \pm}-$те же, что и в $(4.7),(4.8)$, но произведение $\varepsilon \lambda$ нужно заменить на $\delta$. Подчеркнем, что пределом при $\delta \rightarrow 0$ выражения (5.67) служит $\left(1-\chi\left(x_{2} R^{-1}\right)\right) \stackrel{\circ}{2}_{2}^{-}$. Более того, прямые вычисления убеждают нас в том, что найдется $\delta_{2}>0$ такое, что

$$
\left\|\mathbf{u}_{j, \delta}^{-}-\left(1-\chi\left(x_{2} R^{-1}\right)\right) \stackrel{\circ}{u}_{j}^{-} ; \mathcal{V}_{\beta, l}^{l+1}\left(\Pi(\mu) \cap\left\{R<x_{2}<2 R\right\} ; \alpha\right)\right\| \leqslant C \delta^{2} \text { при } \delta<\delta_{2} \text {. }
$$

Определим пространство функций, удовлетворяющих искусственньп условиям излучения. Положим

$$
\mathbf{W}_{\beta, l}^{l+1}(\Pi(\mu) ; \alpha, \delta)=\mathcal{V}_{\beta, l}^{l+1}(\Pi(\mu) ; \alpha) \dot{+} \mathscr{L}\left(\mathbf{u}_{1, \delta}^{-}, \mathbf{u}_{2, \delta}^{-}\right),
$$

где $\mathscr{L}\left(\mathbf{u}_{1, \delta}^{-}, \mathbf{u}_{2, \delta}^{-}\right)$- линеал, натянутый на функции $\mathbf{u}_{1, \delta}^{-}$и $\mathbf{u}_{2, \delta}^{-}$. Таким образом, элементами пространства $\mathbf{W}_{\beta, l}^{l+1}(\Pi(\mu) ; \alpha, \delta)$ являются функции, представимые в виде

$$
u=c_{1} \mathbf{u}_{1, \delta}^{-}+c_{2} \mathbf{u}_{2, \delta}^{-}+u^{\prime},
$$


где $u^{\prime} \in \mathcal{V}_{\beta, l}^{l+1}(\Pi(\mu) ; \alpha)$. Норма в $\mathbf{W}_{\beta, l}^{l+1}(\Pi(\mu) ; \alpha, \delta)$ задается формулой

$$
\left\|u ; \mathbf{W}_{\beta, l}^{l+1}(\Pi(\mu) ; \alpha, \delta)\right\|=\left(\sum_{j=1}^{2}\left|c_{j}\right|^{2}+\left\|u^{\prime} ; \mathscr{V}_{\beta, l}^{l+1}(\Pi(\mu) ; \alpha)\right\|\right)^{1 / 2}
$$

а $c_{1}, c_{2}$ и $u^{\prime}$ берутся из (5.71).

Пусть $\delta<\delta_{2}$; тогда для нормы оператора

$$
\mathbf{L}(\mu, \delta): \mathbf{W}_{\beta, l}^{l+1}(\Pi(\mu) ; \alpha, \delta) \rightarrow \mathcal{V}_{\beta, l}^{l-1}(\Pi(\mu) ; \alpha) \times \mathcal{V}_{\beta, l}^{l-1 / 2}(\Gamma(\mu) ; \alpha),
$$

действуюшего по правилу

$$
\mathbf{L}(\mu, \delta) u=\left\{L_{\delta}(\nabla) u,\left.\partial_{n} u\right|_{\Gamma(\mu)}\right\}
$$

справедливо неравенство

$$
\left\|\mathbf{L}(\mu, \delta): \mathbf{W}_{\beta, l}^{l+1}(\Pi(\mu) ; \alpha, \delta) \rightarrow \mathcal{V}_{\beta, l}^{l-1}(\Pi(\mu) ; \alpha) \times \mathcal{V}_{\beta, l}^{l-1 / 2}(\Gamma(\mu) ; \alpha)\right\| \leqslant C_{\delta_{2}}
$$

Лемма 5.6. Найдутся постоянные $\mu_{2}, C$ такие, что при $\mu<\mu_{2}, \delta<$ $C^{-1} \mu^{\gamma / 2}, \gamma \in(0,1)$, существует оператор $\mathbf{R}(\mu, \delta)$, обратныи й $\kappa$ оператору $\mathbf{L}(\mu, \delta)$, и верна оценка

$$
\left\|\mathbf{R}(\mu, \delta): \mathscr{V}_{\beta, l}^{l-1}(\Pi(\mu) ; \alpha) \times \mathscr{V}_{\beta, l}^{l-1 / 2}(\Gamma(\mu) ; \alpha) \rightarrow \mathbf{W}_{\beta, l}^{l+1}(\Pi(\mu) ; \alpha, \delta)\right\| \leqslant C_{\gamma} \mu^{-\gamma}
$$

в которой постоянная $C_{\gamma}$ не зависит от точек $x^{1}, x^{2}$ и параметров $\delta, \mu$.

ДоказАТЕЛьство. Рассмотрим задачу

$$
\mathbf{L}(\mu, \delta) u=\{f, g\}
$$

где $\{f, g\} \in \mathcal{V}_{\beta, l}^{l-1}(\Pi(\mu) ; \alpha) \times \mathcal{V}_{\beta, l}^{l-1 / 2}(\Gamma(\mu) ; \alpha)$. Операторы $\mathbf{L}(\mu, \delta)$ и $\mathfrak{L}(\mu, \delta)$ неразличимы при $\delta=0$ (функции $\mathbf{u}_{1, \delta}^{-}$и $\mathbf{u}_{2, \delta}^{-}$доопределяются по непрерывности при $\delta=0$; см. (5.69)). Следовательно, лемма 5.5 дает нам решение задачи (5.75) при $\delta=0$. Обозначим его через $\mathbf{R}(\mu, 0)\{f, g\}$. Оно представимо в такой форме:

$$
\mathbf{R}(\mu, 0)\{f, g\}=c_{1}\{f, g\} \mathbf{u}_{1,0}^{-}+c_{2}\{f, g\} \mathbf{u}_{2,0}^{-}+\mathbf{R}^{\prime}(\mu, 0)\{f, g\}
$$

при этом выполнена оценка

$$
\begin{gathered}
\left|c_{1}\{f, g\}\right|^{2}+\left|c_{2}\{f, g\}\right|^{2}+\left\|\mathbf{R}^{\prime}(\mu, 0)\{f, g\} ; \mathscr{V}_{\beta, l}^{l+1}(\Pi(\mu) ; \alpha)\right\|^{2} \\
\leqslant C \mu^{-2 \gamma}\left\|\{f, g\} ; \mathscr{V}_{\beta, l}^{l-1}(\Pi(\mu) ; \alpha) \times \mathcal{V}_{\beta, l}^{l-1 / 2}(\Gamma(\mu) ; \alpha)\right\|^{2}
\end{gathered}
$$


Определим оператор $\widetilde{\mathbf{R}}(\mu, \delta)$, представляюший приближенное решение задачи (5.75) при положительных $\delta$, по формуле

$$
\widetilde{\mathbf{R}}(\mu, \delta)\{f, g\}=c_{1}\{f, g\} \mathbf{u}_{1, \delta}^{-}+c_{2}\{f, g\} \mathbf{u}_{2, \delta}^{-}+\mathbf{R}^{\prime}(\mu, 0)\{f, g\} .
$$

Оценка (5.77) гарантирует непрерывность этого оператора, а неравенства (5.69) и (5.77) показывают, что

$$
\begin{gathered}
\mathbf{L}(\mu, \delta) \widetilde{\mathbf{R}}(\mu, \delta)=\mathbf{1}+\mathscr{S}(\mu, \delta), \\
\left\|\mathscr{S}(\mu, \delta): \mathscr{V}_{\beta, l}^{l-1}(\Pi(\mu) ; \alpha) \times \mathscr{V}_{\beta, l}^{l-1 / 2}(\Gamma(\mu) ; \alpha) \rightarrow \mathscr{V}_{\beta, l}^{l-1}(\Pi(\mu) ; \alpha) \times \mathscr{V}_{\beta, l}^{l-1 / 2}(\Gamma(\mu) ; \alpha)\right\| \\
\leqslant C \mu^{-\gamma} \delta^{2}
\end{gathered}
$$

$\left(\mathbf{1}\right.$ - тождественньй оператор на $\left.\mathscr{V}_{\beta, l}^{l-1}(\Pi(\mu) ; \alpha) \times \mathscr{V}_{\beta, l}^{l-1 / 2}(\Gamma(\mu) ; \alpha)\right)$.

Остается положить

$$
\mathbf{R}(\mu, \delta)=\widetilde{\mathbf{R}}(\mu, \delta)(\mathbf{1}+\mathscr{S}(\mu, \delta))^{-1},
$$

где обратный оператор понимается как ряд Неймана, а неравенство (5.79) и условия леммы гарантируют его сходимость.

Перейдем к построению оператора $\mathfrak{R}(\mu, \delta)$, решаюшего задачу $\mathfrak{L}(\mu, \delta)=\{f, g\}$ в нужном пространстве $\mathbb{W}_{\beta, l}^{l+1}(\Pi(\mu) ; \alpha, \delta)$. Оператор $\mathbf{R}(\mu, \delta)$ также решает эту задачу, но в пространстве $\mathbf{W}_{\beta, l}^{l+1}(\Pi(\mu) ; \alpha, \delta)$. Иными словами, для любых $\{f, g\} \in$ $\mathcal{V}_{\beta, l}^{l-1}(\Pi(\mu) ; \alpha) \times \mathcal{V}_{\beta, l}^{l-1 / 2}(\Gamma(\mu) ; \alpha)$ выполнены соотношения

$$
\begin{aligned}
L_{\delta}(\nabla) \mathbf{R}(\mu, \delta)\{f, g\}=f & \text { на } \Pi(\mu), \\
\partial_{n} \mathbf{R}(\mu, \delta)\{f, g\} & =g \quad \text { на } \Gamma(\mu)
\end{aligned}
$$

и представление на бесконечности

$$
\mathbf{R}(\mu, \delta)\{f, g\}=c_{1}\{f, g\} \mathbf{u}_{1, \delta}^{-}+c_{2}\{f, g\} \mathbf{u}_{2, \delta}^{-}+\mathbf{R}^{\prime}(\mu, \delta)\{f, g\} ;
$$

здесь $\mathbf{R}^{\prime}(\mu, \delta)\{f, g\} \in \mathscr{V}_{\beta, l}^{l+1}(\Pi(\mu) ; \alpha)$. Теорема 2.11 указывает решение $Y_{2}$ однородной задачи (5.49), (5.50), которое может быть представлено как сумма

$$
Y_{2}=u_{2}^{+}+S_{2,1} u_{1}^{-}+S_{2,2} u_{2}^{-}+Y_{2}^{\prime}
$$

с остатком $Y_{2}^{\prime} \in \mathscr{V}_{\beta, l}^{l+1}(\Pi(\mu) ; \alpha)$. Определим оператор $\mathfrak{R}(\mu, \delta)$ по формуле

$$
\mathfrak{R}(\mu, \delta)\{f, g\}=\mathbf{R}(\mu, \delta)\{f, g\}-c_{2}\{f, g\} \frac{1+i}{\sqrt{8}}\left(\delta-\delta^{-1}\right) Y_{2}^{-} .
$$

Из (5.67), (5.68), (5.82) и (5.83) вытекает корректность этого определения. В самом деле, (5.84) может быть переписано в виде

$$
\mathfrak{R}(\mu, \delta)\{f, g\}=\tilde{c}_{1}\{f, g\} u_{1}^{-}+\tilde{c}_{2}\{f, g\} u_{2}^{-}+\mathfrak{R}^{\prime}(\mu, \delta)\{f, g\},
$$


где согласно (5.82)

$$
\begin{gathered}
\tilde{c}_{1}\{f, g\}=c_{1}\{f, g\}-c_{2}\{f, g\} \frac{1+i}{\sqrt{8}}\left(\delta-\delta^{-1}\right) S_{2,1}, \\
\tilde{c}_{2}\{f, g\}=-i c_{2}\{f, g\} \frac{1+i}{\sqrt{8}}\left(\delta+\delta^{-1}\right)-c_{2}\{f, g\} \frac{1+i}{\sqrt{8}}\left(\delta-\delta^{-1}\right) S_{2,2}, \\
\mathfrak{R}^{\prime}(\mu, \delta)\{f, g\}=\mathbf{R}^{\prime}(\mu, \delta)\{f, g\}+c_{2}\{f, g\} \frac{1+i}{\sqrt{8}}\left(\delta-\delta^{-1}\right) Y_{2}^{\prime} .
\end{gathered}
$$

Из (5.86), (5.87) и леммы 5.6 следует, что

$$
\left|\tilde{c}_{j}\right| \leqslant C \delta^{-1} \mu^{-\gamma}\left\|\{f, g\} ; \mathscr{V}_{\beta, l}^{l-1}(\Pi(\mu) ; \alpha) \times \mathscr{V}_{\beta, l}^{l-1 / 2}(\Gamma(\mu) ; \alpha)\right\|, \quad j=1,2 .
$$

Оценим норму $Y_{2}^{\prime} \in \mathcal{V}_{\beta, l}^{l+1}(\Pi(\mu) ; \alpha)$. В силу теоремы 2.11 и формулы $(5.83)$

$$
\begin{array}{lr}
L_{\delta}(\nabla) Y_{2}^{\prime}=0 & \text { на } \Pi(\mu), \\
\partial_{n} Y_{2}^{\prime}=-\partial_{n}\left(u_{2}^{+}+S_{2,1} u_{1}^{-}+S_{2,2} u_{2}^{-}\right) & \text {на } \quad \Gamma(\mu) .
\end{array}
$$

Лемма 5.6 и определения (4.7), (4.8) влекут оценку

$$
\left\|Y_{2}^{\prime} ; \mathscr{V}_{\beta, l}^{l+1}(\Pi(\mu) ; \alpha)\right\| \leqslant C \delta^{-1}
$$

Собирая (5.74), (5.89) и (5.92), убеждаемся в справедливости следующей теоремы.

ТЕОРема 5.7. Найдутся такие постоянные $\mu_{3}, C$, что при $\mu<\mu_{3}$, $\delta<C \mu^{\gamma / 2}, \gamma \in(0,1)$, существует оператор $\mathfrak{R}(\mu, \delta)$, обратный $\kappa$ оператору $\mathfrak{L}(\mu, \delta)$ :

$$
\left\|\Re(\mu, \delta): \mathcal{V}_{\beta, l}^{l-1}(\Pi(\mu) ; \alpha) \times \mathcal{V}_{\beta, l}^{l-1 / 2}(\Gamma(\mu) ; \alpha) \rightarrow \mathbb{W}_{\beta, l}^{l+1}(\Pi(\mu) ; \alpha, \delta)\right\| \leqslant C \mu^{-\gamma} \delta^{-2} .
$$

Постоянная $C$ не зависит от точек $x^{1}, x^{2}$ и параметров $\delta$ u $\mu$, причем для коэффициентов при уходящих волнах справедливы более сильные оцен$\kappa u(5.89)$.

ЗАмЕЧАНИЕ 5.8. Для доказательства предложения 4.2 требуется положить $\delta=\lambda \mu$ при $\lambda \in\left[\frac{1}{2} k_{0}^{2}, 2 k_{0}^{2}\right]$. В этом случае остаются справедливыми аналогичные (5.89) и (5.93) оценки с постоянной $C$, не зависящей от $\lambda, \mu$ и $x^{1}, x^{2}$.

ЗАмЕчАНИЕ 5.9. В $\S 3$ мы построили асимптотические разложения матриц рассеяния при фиксированной форме периодической кривой. На заключительном этапе доказательства теоремы 5.7 (см. п. 3) специальная геометрия границы по существу востребована не была - использовалось лишь наличие оценки (5.66) для нормы оператора, обратного к предельному оператору $\mathfrak{L}(\mu, 0)$ (т.е. оператора, действующего на пороге). В условиях $\S 3$ аналогичная оценка конечно же существует (см. следствие 2.8), а значит, теорема 5.7 обосновывает выведенные в $\S 3$ формулы.

ЗАмЕчАнИЕ 5.10. В п. $7 \S 3$ мы привели формулы для коэффициентов в асимптотическом разложении матрицы рассеяния в послепороговой ситуации. Процедура их обоснования аналогична способу, описанному в п. 3. 


\section{Список литературы}

1. Камочкий И. В., Назаров С. А. Аномалии Вуда и поверхностные волны в задаче рассеяния на периодической границе. I // Матем. сб. 1998. Т. 190. №1. С. 109-138.

2. Кондратьев B. A. Краевые задачи для эллиптических уравнений в областях с коническими или угловыми точками // Труды ММО. 1967. Т. 16. С. 209-292.

3. Mazja W. G., Nasarov S.A., Plamenewski B.A. Asymptotishe Theorie elliptischer Randwertaufgaben in singulär gestörten Gebieten. V. 1. Berlin: Akademie-Verlag, 1990.

4. Ильин A. M. Согласование асимптотических разложений решений краевых задач. М.: Наука, 1989.

5. Бабич В. М. О теореме существования решения задач Дирихле и Неймана для уравнения Гельмгольца в квазипериодическом случае // Сиб. матем. журн. 1988. Т. 39. № 2. С. 3-9.

6. Колмогоров A.H., Фомин C. В. Элементы теории функций и функционального анализа. М.: Наука, 1981.

7. Nasarov S. A., Plamenewski B. A. Elliptic problems in domains with piecewise smooth boundaries. Berlin: Walter de Gruyter, 1994.

8. Назаров С. А., Пламеневский Б. А. Эллиптические задачи в областях с кусочно гладкой границей. М.: Наука, 1991.

Санкт-Петербургский государственньй университет;

Государственная морская академия

Поступила в редакцию

им. адм. С. О. Макарова, Санкт-Петербург

08.07.1997 